\title{
Review and Progress towards the Common Broadband Management of High-Voltage Transmission Grids: Model Expansion and Comparative Modal Analysis
}

\author{
Athanasios G. Lazaropoulos \\ School of Electrical and Computer Engineering, National Technical University of Athens, 9 Iroon Polytechniou Street, Zografou, \\ 15780 Athens, Greece \\ Correspondence should be addressed to Athanasios G. Lazaropoulos, aglazaropoulos@gmail.com
}

Received 27 August 2012; Accepted 30 September 2012

Academic Editors: J. Abu Qahouq, C. W. Chiou, and A. Mercha

Copyright () 2012 Athanasios G. Lazaropoulos. This is an open access article distributed under the Creative Commons Attribution License, which permits unrestricted use, distribution, and reproduction in any medium, provided the original work is properly cited.

The need of bridging the digital gap between underdeveloped/developed areas and promoting smart grid (SG) networks urges the deployment of broadband over power lines (BPL) systems and their further integration. The contribution of this paper is fourfold. First, based on the well-established hybrid model of (Lazaropoulos and Cottis 2009, 2010, Lazaropoulos, 2012) and the generic multidimensional network analysis tool presented in (Lazaropoulos 2012, Sartenaer 2004, Sartenaer and Delogne 2006, 2001) an exact multidimensional chain scattering matrix method, which is suitable for overhead high-voltage/broadband over power lines (HV/BPL) networks, is proposed and is evaluated against other theoretical and experimental proven models. Second, the proposed method investigates the overhead HV/BPL transmission grids (overhead $150 \mathrm{kV}$ single-circuit, $275 \mathrm{kV}$ double-circuit, and $400 \mathrm{kV}$ double-circuit multiconductor structures) with regard to their end-to-end signal attenuation. It is found that the above features depend drastically on the overhead power grid type, the frequency, the MTL configuration, the physical properties of the cables used, the end-to-end distance, and the number, the length, and the terminations of the branches encountered along the end-toend BPL signal propagation. Third, the impact of the multiplicity of the branches at the same junction in overhead HV grids is first examined. Based on the inherent long-branch structure and the quasi-static behavior of single/multiple branches with matched terminations of overhead HV grid, a simple approach suitable for overhead HV/BPL channel estimation is presented. Fourth, identifying the similar characteristics among different overhead HV/BPL configurations, an additional step towards the common overhead HV/BPL analysis is demonstrated; the entire overhead HV/BPL grid may be examined under a common PHY framework regardless of the overhead HV/BPL grid type examined. Finally, apart from the presentation of broadband transmission potential of the entire overhead transmission power grid, a consequence of this paper is that it helps towards: (i) the better broadband monitoring and management of overhead HV transmission power grids in an interactive SG network; and (ii) the intraoperability/interoperability of overhead HV/BPL systems under the aegis of a unified transmission/distribution SG power network.

\section{Introduction}

Due to ubiquitous nature of the transmission and distribution power grids, the structure of these grids-that is, low-voltage (LV), medium-voltage (MV), and high-voltage (HV) grids-is the key to developing an IP-based power system, offering a plethora of potential smart grid (SG) applications [1-5]. In the meanwhile, the deployment of broadband over power lines (BPL) networks through the entire transmission and distribution grid forms a potentially convenient and inexpensive communications medium for delivering broadband last mile access in remote and/or underdeveloped areas [6-8].

Since overhead HV power lines are generally the lowest cost method of transmission for large quantities of electric energy, utilities mainly employ overhead HV power grids for new urban, suburban, and rural installations [9-16]. Apart from the power delivery, the availability of a reliable communication network on the HV power grid side is important for the support of the significant changes towards upcoming 
SG transformations in transmission power networks $[9,17-$ 20].

Since the overhead power grid was not originally designed to serve the purpose of a transmission medium for communication signals, the overhead transmission power grids are subjected to attenuation, multipath due to various reflections, noise, and electromagnetic interference [1-3, 21-31]. Each of the aforementioned adverse factors affects critically the overall performance and the design of BPL systems [32-35].

Due to the need of broadband communications through transmission power grids, the development of accurate channel models at high frequencies along the overhead HV transmission power lines is imperative. As usually done in BPL transmission, a hybrid model is employed to examine the behavior of BPL transmission channels installed on BPL multiconductor transmission line (MTL) structures $[1,2,21-25,36-41]$. Through a bottom-up approach consisting of an appropriate combination of the similarity transformation and MTL theory, the modes that may be supported by an overhead HV/BPL configuration are determined concerning their propagation constants and their characteristic impedances [1-3, 21-25, 35-47]. Based on $[3,36,42,43]$, in this paper, a top-down approach is proposed to determine the end-to-end attenuation of overhead HV/BPL channels based on an exact version of multidimensional chain scattering matrix or T-Matrix method. This novel extended chain scattering matrix or TMatrix (TM2) method, which is already well verified in the case of overhead and underground LV/BPL and MV/BPL distribution power grids [3], combines the accuracy of the generic multidimensional network analysis tool presented in $[3,36,42,43]$ and the simplicity of hybrid model of [1$3,21-25]$. Based on numerical results concerning various simulated overhead HV/BPL topologies, BPL transmission via the overhead transmission power grid is investigated aiming at clarifying the influence of factors, such as the overhead HV power grid type $(150 \mathrm{kV}, 275 \mathrm{kV}$, or $400 \mathrm{kV}$ and single or double circuit), the physical properties of the cables used, the MTL configuration, the end-to-end distance, and the number, the length, the terminations, and the multiplicity of the branches encountered along the endto-end BPL signal propagation.

Through the exhaustive comparative analysis of a great number of overhead transmission power grid numerical results, the common nature of overhead HV/BPL systems is disclosed resulting in a common PHY framework as it concerns the BPL signal transmission through their power lines. Combining these special BPL signal transmission characteristics with the quasi-static spectral behavior of single/multiple branches with matched terminations, a simplified channel modeling approach suitable for overhead HV/BPL networks is proposed.

Following the first steps towards overhead HV/BPL system integration made in $[1,2]$, a consequence of the proposed top-down modeling and common PHY framework is that their combination further enhances the intraoperability/interoperability of overhead HV/BPL systems in a SG environment.
The rest of this paper is organized as follows. In Section 2, the overhead HV configurations adopted in this paper are demonstrated. Section 3 resumes the MTL theory and eigenvalue decomposition (EVD) modal analysis concerning overhead HV/BPL transmission. Section 4 deals with signal transmission via overhead HV power lines by the proposed TM2 method which is applied for the evaluation of the end-to-end EVD modal transfer functions. In Section 5, numerical results are provided, aiming at marking out the validity of TM2 method compared to other well-proven models and how the various features of the overhead transmission power grids influence BPL transmission. On the basis of these numerical results, the common PHY framework of overhead HV/BPL systems is further enriched. Section 5 recapitulates the conclusions of this paper.

\section{Overhead HV Transmission Power Networks}

The overhead HV power grid differs considerably from transmission via twisted-pair, coaxial, or fiber-optic cables due to the idiosyncrasy of the network structure and the physical properties of the power cables used $[1,2,10,11,21$, $23,26,29,38,48]$.

According to [2], the electrical power industry classifies overhead HV power transmission systems into three main categories, depending on: (i) the voltage levels (from $150 \mathrm{kV}$ up to $1000 \mathrm{kV})$, (ii) the number of MTL circuits per each tower (mainly, either single or double circuit); in the case of single-circuit three-phase overhead HV systems, each tower supports three-phase conductors whereas in the case of double-circuit three-phase overhead HV systems, each tower supports six-phase conductors; (iii) the number of neutral conductors per each tower $[1,2,15,16,49,50]$.

A typical case of $150 \mathrm{kV}$ single-circuit overhead HV transmission line is depicted in Figure 1(a). Three parallel phase conductors spaced by $\Delta_{p}^{150 \mathrm{kV}}$ are suspended at heights $h_{p}^{150 \mathrm{kV}}$ above lossy ground-conductors 1, 2, and 3 . Moreover, two parallel neutral conductors spaced by $\Delta_{n}^{150 \mathrm{kV}}$ hang at heights $h_{n}^{150 \mathrm{kV}}$ - conductors 4 and 5-(for more details see [2]). This three-phase five-conductor $\left(n^{150 \mathrm{kV}}=5\right)$ overhead HV distribution line configuration is considered in the present work consisting of ACSR GROSBEK $3 \times$ $374.77 \mathrm{~mm}^{2}+2 \times 322.26 \mathrm{~mm}^{2}$ conductors $[1,2,14-16,51-$ $54]$.

A typical case of overhead $275 \mathrm{kV}$ double-circuit overhead HV transmission line is illustrated in Figure 1(b). Two sets of three phase conductors-conductors 1, 2, 3 and conductors 4, 5, 6-are spaced by $\Delta_{p 1}^{275 \mathrm{kV}}$. The three phase conductors of each set are suspended one above the other spaced by $\Delta_{p 2}^{275 \mathrm{kV}}$ and located at heights $h_{p}^{275 \mathrm{kV}}$ above ground for the lowest conductor. Apart from the six phase conductors of two sets, the upper conductor-conductor 7 -is the neutral conductor with radii $r_{n}^{275 \mathrm{kV}}$, spaced by $\Delta_{n}^{275 \mathrm{kV}}$ from the highest phase conductor-conductor 3 or 6-, and, consequently, hang at heights $h_{n}^{275 \mathrm{kV}}$ above ground (for more details see [2]). This double-circuit sevenconductor $\left(n^{275 \mathrm{kV}}=7\right)$ overhead HV distribution line configuration is considered in the present work consisting of ACSR conductors [1, 2, 14-16, 51-57]. 


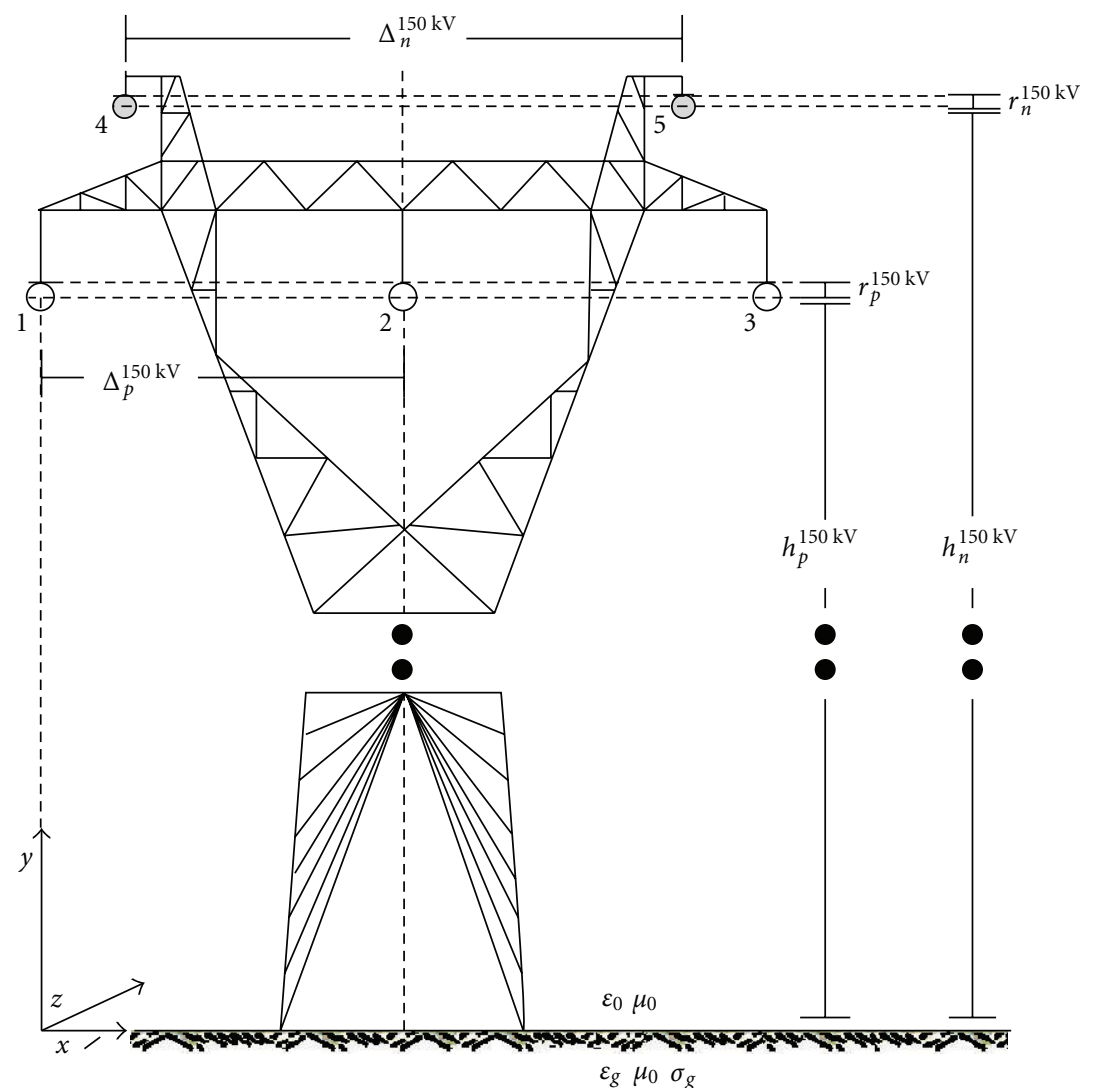

(a)

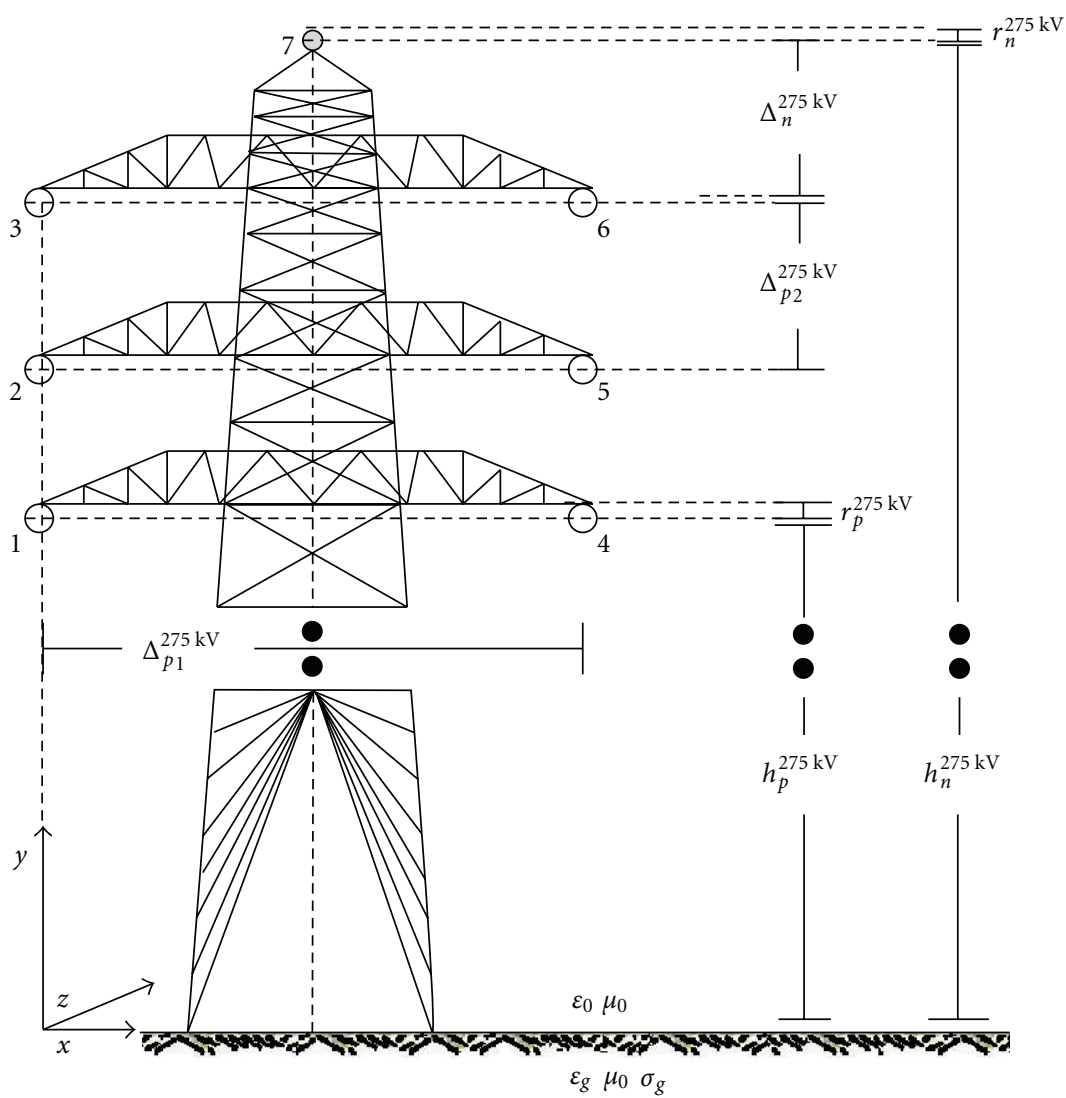

(b)

FIgURE 1: Continued. 


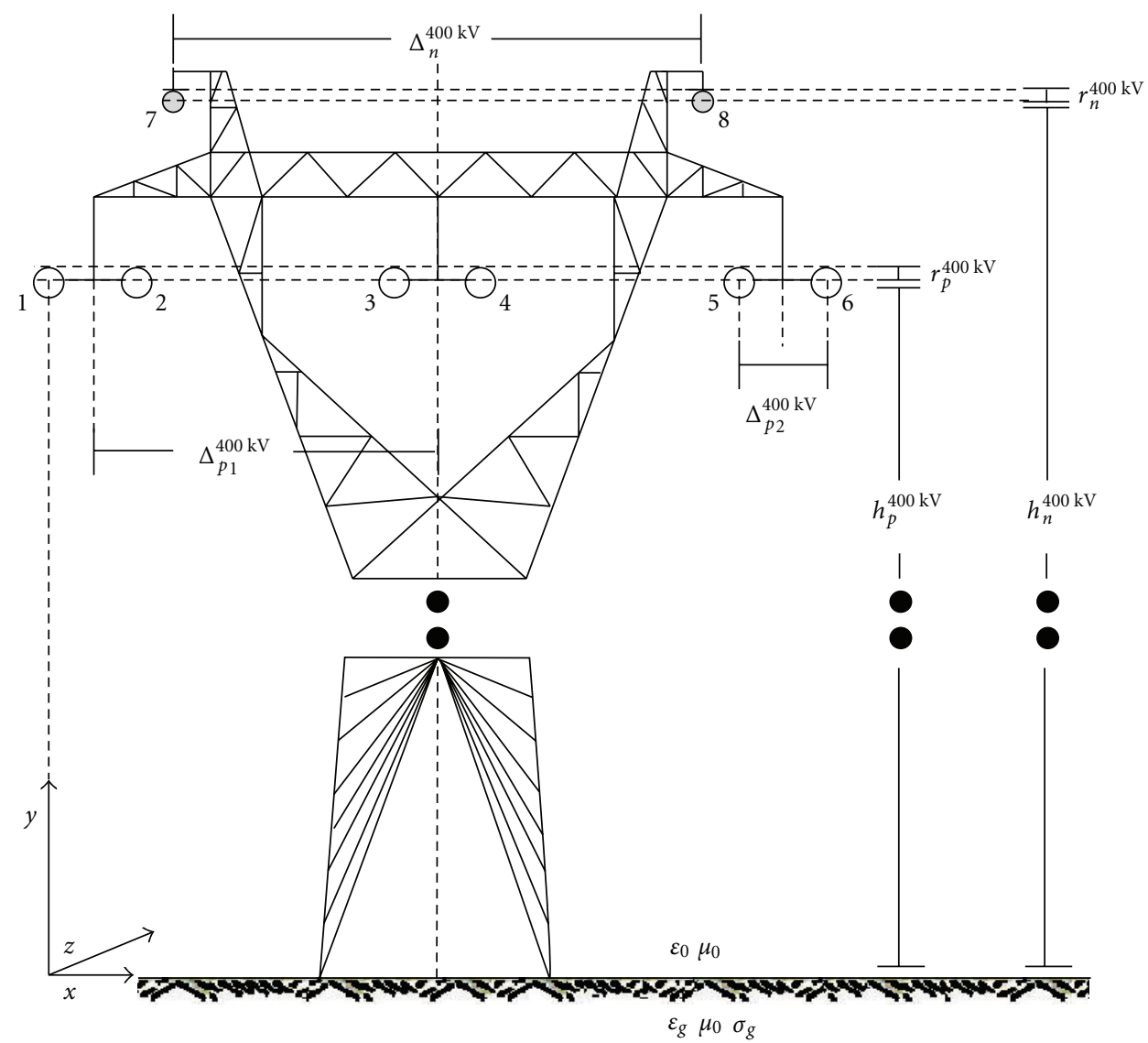

(c)

Figure 1: Typical overhead HV multiconductor structures [1, 2, 14, 51-54]. (a) $150 \mathrm{kV}$ single circuit. (b) $275 \mathrm{kV}$ double circuit. (c) $400 \mathrm{kV}$ double circuit.

Overhead $400 \mathrm{kV}$ double-circuit overhead HV transmission phase lines with radii $r_{p}^{400 \mathrm{kV}}$ hang at typical heights $h_{p}^{400 \mathrm{kV}}$ above ground-conductors 1, 2, 3, 4, 5, and 6 . These six phase conductors are divided into three bundles; the phase conductors of each bundle are connected by nonconducting spacers and are separated by $\Delta_{p 2}^{400 \mathrm{kV}}$, whereas bundles are spaced by $\Delta_{p 1}^{400 \mathrm{kV}}$. Moreover, two parallel neutral conductors with radii $r_{n}^{400 \mathrm{kV}}$ spaced by $\Delta_{n}^{400 \mathrm{kV}}$ hang at heights

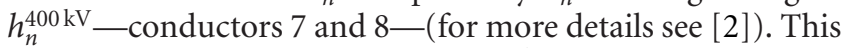
double-circuit eight-conductor $\left(n^{400 \mathrm{kV}}=8\right)$ overhead HV distribution line configuration is considered in the present work consisting of ACSR conductors-see Figure 1(c) $[1,2,14-16,49-54]$.

The ground is considered as the reference conductor. The conductivity of the ground is assumed $\sigma_{g}=5 \mathrm{mS} / \mathrm{m}$ and its relative permittivity $\varepsilon_{r g}=13$, which is a realistic scenario [ 1 , $2,21,22,38,48]$. The impact of imperfect ground on signal propagation via overhead power lines was analyzed in [21, $22,38,48,58-65]$.

\section{MTL Theory and EVD Modal Analysis}

Compared to a two-conductor line supporting one forwardand one backward-traveling wave, an MTL structure with
$n^{X V}+1$ conductors parallel to the $z$ axis as depicted in Figures $1(\mathrm{a})-1(\mathrm{c})$ may support $n^{X V}$ pairs of forward- and backward-traveling waves with corresponding propagation constants where $[\cdot]^{X V}$ denotes the overhead HV/BPL grid type considered-for example, $150 \mathrm{kV}, 275 \mathrm{kV}$, and $400 \mathrm{kV}$ in the case of $150 \mathrm{kV}$ single-circuit, $275 \mathrm{kV}$ double-circuit, and $400 \mathrm{kV}$ double-circuit overhead HV/BPL configuration, respectively. These waves may be described by a coupled set of $2 n^{X V}$ first-order partial differential equations relating the line voltages $V_{i}(z, t), i=1, \ldots, n^{X V}$, to the line currents $I_{i}(z, t), i=1, \ldots, n^{X V}$, while each pair of forward- and backward-traveling waves is referred to as a mode $[1-3,21$, $23,44,45]$.

Consequently, in the case of overhead HV transmission lines involving $n^{X V}$ conductors over lossy plane ground, $n^{X V}$ modes may be supported, namely, $[1-4,9,10,13,14,21,36$, $38,39,42-45,48,51,52,58-61,66-68]$.

(1) Common mode of overhead BPL transmission $\left(\mathrm{CM}^{X V}\right)$ which propagates via the $n^{X V}$ conductors and returns via the ground. $\gamma_{\mathrm{CM}}^{X V} \equiv \gamma_{1}^{X V}$ constitutes the CMXV propagation constant. The spectral behavior of $\mathrm{CM}^{150 \mathrm{kV}}, \mathrm{CM}^{275 \mathrm{kV}}$, and $\mathrm{CM}^{400 \mathrm{kV}}$ is thoroughly investigated in $[1,2]$. 
(2) Differential modes of overhead BPL transmission $\left(\mathrm{DM}_{i}^{X V}, i=1, \ldots, n^{X V}-1\right)$ which propagate and return via the $n^{X V}$ conductors. $\gamma_{\mathrm{DM} i}^{X V} \equiv \gamma_{i+1}^{X V}, i=$ $1, \ldots, n^{X V}-1$, constitute the propagation constants of $\mathrm{DM}_{i}^{X V}, i=1, \ldots, n^{X V}-1$, respectively. The spectral behavior of $\mathrm{DM}^{150 \mathrm{kV}} \mathrm{s}, \mathrm{DM}^{275 \mathrm{kV}} \mathrm{s}$, and $\mathrm{DM}^{400 \mathrm{kV}} \mathrm{s}$ is thoroughly investigated in $[1,2]$.

As it has already been presented in [1-3, 21-25], the EVD modal voltages $\mathbf{V}^{X V, m}(z)=\left[V_{1}^{X V, m}(z) \cdots\right.$ $\left.V_{n}^{X V, m}(z)\right]^{\mathrm{T}}$ and the EVD modal currents $\mathbf{I}^{X V, m}(z)=$ $\left[I_{1}^{X V, m}(z) \cdots I_{n}^{X V, m}(z)\right]^{\mathrm{T}}$ may be related to the respective line quantities $\mathbf{V}^{X V}(z)=\left[V_{1}^{X V}(z) \cdots V_{n}^{X V}(z)\right]^{\mathrm{T}}$ and $\mathbf{I}^{X V}(z)=$ $\left[I_{1}^{X V}(z) \cdots I_{n}^{X V}(z)\right]^{\mathrm{T}}$ via the similarity transformations [21, $23,36,44,45]$

$$
\begin{gathered}
\mathbf{V}^{X V}(z)=\mathbf{T}_{V}^{X V} \cdot \mathbf{V}^{X V, m}(z), \\
\mathbf{I}^{X V}(z)=\mathbf{T}_{\mathrm{I}}^{X V} \cdot \mathbf{I}^{X V, m}(z),
\end{gathered}
$$

where $[\cdot]^{\mathrm{T}}$ denotes the transpose of a matrix; $\mathbf{T}_{V}^{X V}$ and $\mathbf{T}_{I}^{X V}$ are $n^{X V} \times n^{X V}$ matrices depending on the overhead power grid type, the frequency, the physical properties of the cables, and the geometry of the MTL configuration $[3,9,13,14$, $21,23,36,44,45,51,52,67,68]$. It is assumed that $\mathbf{U}^{X V}=$ $\left(\mathbf{T}_{V}^{X V} \mathbf{T}_{I}^{X V}\right)$ is the eigenbase of the MTL configuration.

Through the aforementioned equations, the line voltages and currents are expressed as appropriate superpositions of the respective EVD modal quantities. From (1)

$$
\mathbf{V}^{X V, m}(0)=\left[\mathbf{T}_{V}^{X V}\right]^{-1} \cdot \mathbf{V}^{X V}(0)
$$

The TM2 method-presented in Section 3-models the spectral relationship between $V_{i}^{X V, m}(z), i=1, \ldots, n^{X V}$, and $V_{j}^{X V, m}(0), j=1, \ldots, n^{X V}$, proposing operators $H_{i, j}^{X V, m}\{\cdot\}$, $i, j=1, \ldots, n^{X V}$, so that [3]

$$
\mathbf{V}^{X V, m}(z)=\mathbf{H}^{X V, m}\left\{\mathbf{V}^{X V, m}(0)\right\},
$$

where $\mathbf{H}^{X V, m}\{\cdot\}$ is the $n^{X V} \times n^{X V}$ EVD modal transfer function matrix whose elements $H_{i, j}^{X V, m}\{\cdot\}, i, j=1, \ldots, n^{X V}$, with $i=j$ are the EVD modal cochannel (CC) transfer functions, while those $H_{i, j}^{X V, m}\{\cdot\}, i, j=1, \ldots, n^{X V}$ with $i \neq j$ are the EVD modal cross-channel (XC) transfer functions and $H_{i, j}^{X V, m}$ denotes the element of matrix $\mathbf{H}^{X V, m}\{\cdot\}$ in row $i$ of column $j[1-3,21-23,25]$.

Combining (1) and (4), the $n^{X V} \times n^{X V}$ transfer function matrix $\mathbf{H}^{X V}\{\cdot\}$ relating $\mathbf{V}^{X V}(z)$ with $\mathbf{V}^{X V}(0)$ through

$$
\mathbf{V}^{X V}(z)=\mathbf{H}^{X V}\left\{\mathbf{V}^{X V}(0)\right\}
$$

is determined from

$$
\mathbf{H}^{X V}\{\cdot\}=\mathbf{T}_{V}^{X V} \cdot \mathbf{H}^{X V, m}\{\cdot\} \cdot\left[\mathbf{T}_{V}^{X V}\right]^{-1} .
$$

Based on (6), the $n^{X V} \times n^{X V}$ transfer function matrix $\mathbf{H}^{X V}\{\cdot\}$ of each overhead HV/BPL transmission networks is determined [1-3, 21-25, 39, 66].

\section{Evaluation of the End-to-End EVD Modal Transfer Functions}

So far, several methods with converging results have been used to determine the EVD modal transfer functions of BPL transmission channels $[1-3,21-25,36,39-43,47,66,69$, 70]. In this paper, the TM2 method, which is already well verified in the case of overhead and underground MV/BPL and LV/BPL distribution power grids [3], is applied. TM2 method constitutes a refined exact version of scattering matrix (SM) and TM methods - analyzed in [1-3, 21-25]exploiting the generic multidimensional network analysis tool-presented in $[3,36,42,43]$. This new version of TM method is tailored especially for overhead HV/BPL networks.

More specifically, similarly to TM method, in order to apply the TM2 method, an end-to-end overhead HV/BPL connection is separated into segments-network modules, each of them comprising the successive branches encountered-see Figure 2(a)-.

4.1. Transmission via the Network Modules. In the present analysis, signal transmission through the various network modules is taken into account based on the concatenation of respective chain scattering or $T$-Matrices. A typical overhead HV/BPL end-to-end connection comprises branch-type network modules, as depicted in Figure 2(b), while A and B are assumed matched [1-3, 11, 21-25, 32, 38, 48, 59]. These "branch" modules may be considered as a cascade of two submodules. To determine the multidimensional scattering characteristics of these two submodules, the scattering matrix formalism of the generic multidimensional network analysis tool is adopted [3, 36, 42, 43]. In detail, these two submodules are as follows [3].

(i) A "transmission" submodule representing a transmission line of length $L_{k}$ with the $2 n^{X V, k} \times 2 n^{X V, k}$ scattering matrix

$$
\begin{aligned}
\mathbf{S}^{T, k}\left(\boldsymbol{\gamma}^{X V, k}, L_{k}, \mathbf{U}^{X V, k}\right) & =\left[\begin{array}{ll}
\mathbf{S}_{11}^{T, k} & \mathbf{S}_{12}^{T, k} \\
\mathbf{S}_{21}^{T, k} & \mathbf{S}_{22}^{T, k}
\end{array}\right] \\
& =\left[\begin{array}{cc}
\mathbf{0}_{n^{X V, k} \times n^{X V, k}} & \mathbf{E}^{T, k} \\
\mathbf{E}^{T, k} & \mathbf{0}_{n^{X V, k} \times n^{X V, k}}
\end{array}\right],
\end{aligned}
$$

where $\mathbf{E}^{T, k}=\operatorname{diag}\left\{\exp \left(-\gamma_{1}^{X V, k} L_{k}\right) \cdots \exp \left(-\gamma_{n}^{X V, k} L_{k}\right)\right\}$ is the corresponding $n^{X V, k} \times n^{X V, k}$ diagonal transmission matrix, $\mathbf{0}_{n^{X V, k} \times n^{X V, k}}$ is an $n^{X V, k} \times n^{X V, k}$ matrix with zero elements and $\mathbf{S}_{11}^{T, k}, \mathbf{S}_{12}^{T, k}, \mathbf{S}_{21}^{T, k}, \mathbf{S}_{22}^{T, k}$ are the matrix elements of the $\boldsymbol{S}^{T, k}$ as evaluated from (7). $\gamma^{X V, k}, \mathbf{U}^{X V, k}$, and $n^{X V, k}$ are the set with elements the propagation constant of the modes, the eigenbase, and the number of conductors, respectively. In the cases examined in this paper and as it has previously been applied in [3], since one type of overhead HV/BPL configuration is used across the overhead $\mathrm{HV} / \mathrm{BPL}$ end-to-end connection, it is assumed that $\mathbf{U}^{X V} \equiv \mathbf{U}^{X V, k}$ and $n^{X V}=n^{X V, k}$ are the reference eigenbase and the reference number of conductors, respectively. 
(a)

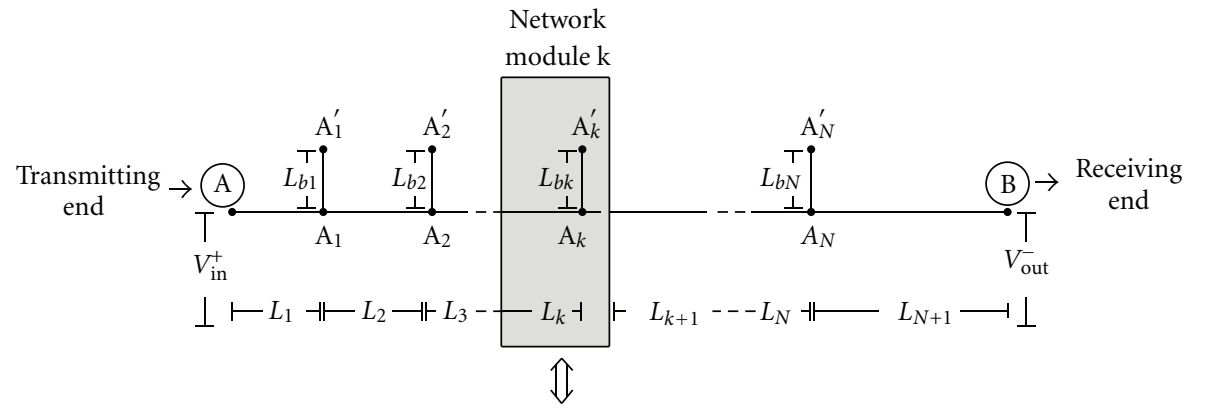

(b)

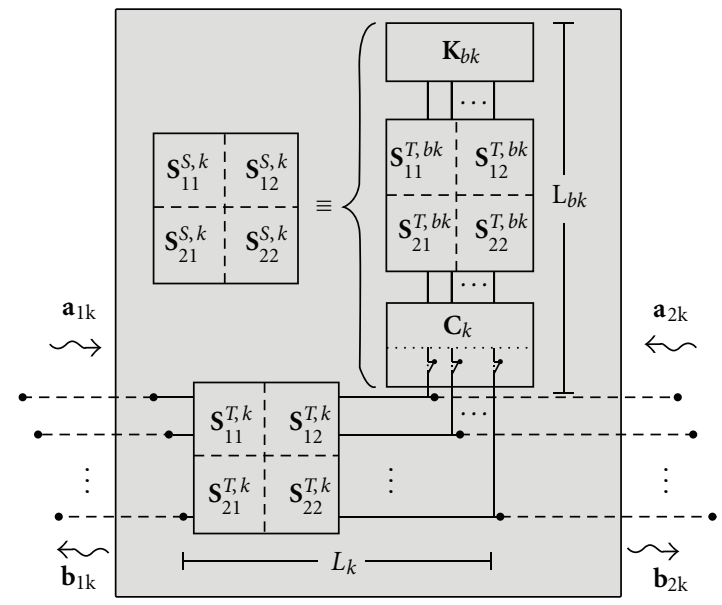

(c)

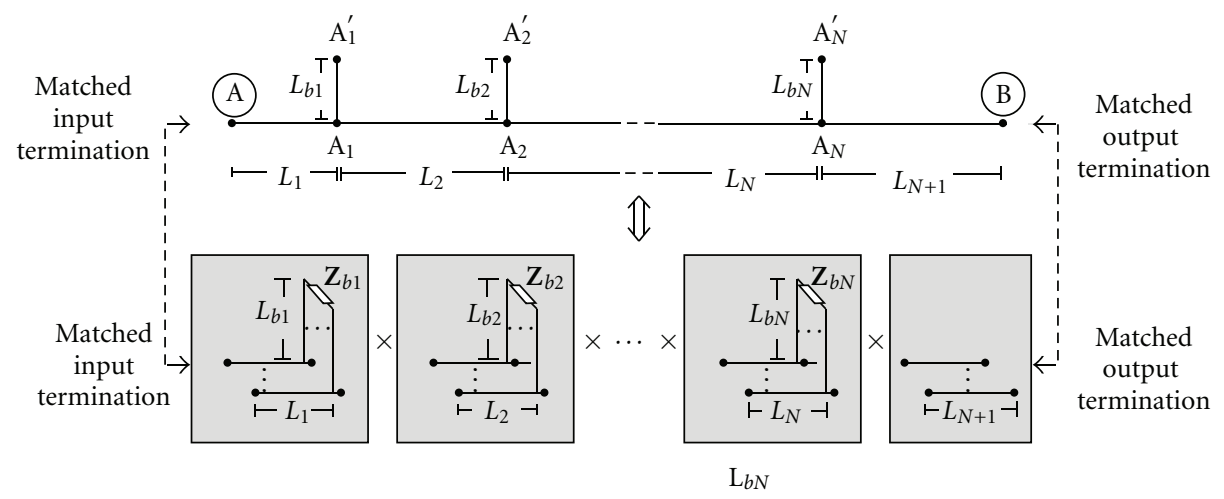

Figure 2: (a) End-to-end HV/BPL connection with $N$ branches. (b) Network module. (c) An indicative HV/BPL topology considered as a cascade of $N+1$ modules corresponding to $N$ branches [1-3, 21, 22, 24, 25].

(ii) A "shunt" submodule representing the cascade of [3]: (a) an $n^{X V, b k} \times n^{X V, b k}$ reflection matrix $\mathbf{K}_{b k}\left(\mathbf{Z}_{b k}\right)$ associated with the branch terminations; (b) an $2 n^{X V, b k} \times 2 n^{X V, b k}$ scattering matrix $\mathbf{S}^{T, b k}\left(\gamma^{X V, b k}, L_{b k}, \mathbf{U}^{X V}\right)$-similar expression to (7)representing the branch transmission line of length $L_{b k}$; (c) an $n^{X V, b k} \times n^{X V, k}$ connection elements $\mathbf{C}_{k}$ defining the interconnections between the transmission and branch conductors; with $2 n^{X V, k} \times 2 n^{X V, k}$ scattering matrix

$$
\mathbf{S}^{S, k}\left(\gamma^{X V, b k}, L_{b k}, \mathbf{U}^{X V}\right)=\left[\begin{array}{ll}
\boldsymbol{S}_{11}^{S, k} & \mathbf{S}_{12}^{S, k} \\
\mathbf{S}_{21}^{S, k} & \mathbf{S}_{22}^{S, k}
\end{array}\right]
$$

where $\mathbf{Z}_{b k}$ is the $n^{X V, b k} \times n^{X V, b k}$ branch termination matrix, $\mathbf{S}_{11}^{S, k}, \boldsymbol{S}_{12}^{S, k}, \boldsymbol{S}_{21}^{S, k}, \mathbf{S}_{22}^{S, k}$ are the matrix elements of the $S^{S, k}$ matrix as determined from (8), and $\gamma^{X V, b k}$ and $n^{X V, b k}$ are the set with elements the propagation constant of the modes and the number of conductors, respectively, related to this "shunt" submodule overhead MTL configuration [3, 36, 42, 43 ]. When the number of conductors varies from "transmission" to "shunt" submodules, through the eigenbase change procedure-presented analytically in $[3,36,42,43]$ - and by taking into consideration the reference eigenbase $\mathbf{U}^{X V}$ and reference number of conductors $n^{X V}$, scattering matrices $\mathbf{K}_{b k}, \mathbf{S}^{T, b k}$, and $\mathrm{C}_{k}$ may be grouped together through their cascading. Hence, the "shunt" scattering matrix of (8) may be evaluated.

Based on $[3,71,72]$ and by using (7) and (8), the $2 n^{X V} \times$ $2 n^{X V}$ "transmission" submodule chain scattering matrix $\mathbf{T}^{T, k}$ 
and the $2 n^{X V} \times 2 n^{X V}$ "shunt" submodule chain scattering matrix $\mathbf{T}^{S, k}$ are determined by [3]

$$
\begin{aligned}
\mathbf{T}^{T, k} & =\left[\begin{array}{cc}
\mathbf{T}_{11}^{T, k} & \mathbf{T}_{12}^{T, k} \\
\mathbf{T}_{21}^{T, k} & \mathbf{T}_{22}^{T, k}
\end{array}\right]=\left[\begin{array}{cc}
{\left[\mathbf{E}^{T, k}\right]^{-1}} & \mathbf{0}_{n^{X V} \times n^{X V}} \\
\mathbf{0}_{n^{X V} \times n^{X V}} & \mathbf{E}^{T, k}
\end{array}\right], \\
\mathbf{T}^{S, k}= & {\left[\begin{array}{cc}
\mathbf{T}_{11}^{S, k} & \mathbf{T}_{12}^{S, k} \\
\mathbf{T}_{21}^{S, k} & \mathbf{T}_{22}^{S, k}
\end{array}\right] } \\
= & {\left[\begin{array}{cc}
{\left[\mathbf{S}_{21}^{S, k}\right.} & -1 \\
{\left[\mathbf{S}_{21}^{S, k}\right]^{-1} \mathbf{S}_{11}^{S, k}} & \mathbf{S}_{12}^{S, k}-\mathbf{S}_{11}^{S, k}\left[\mathbf{S}_{21}^{S, k}\right]^{-1} \mathbf{S}_{22}^{S, k}
\end{array}\right], }
\end{aligned}
$$

where $\mathbf{T}_{11}^{T, k}, \mathbf{T}_{12}^{T, k}, \mathbf{T}_{21}^{T, k}, \mathbf{T}_{22}^{T, k}$ are the $n^{X V} \times n^{X V}$ matrix elements of the $\mathbf{T}^{T, k}$ as determined by (9) and $\mathbf{T}_{11}^{S, k}, \mathbf{T}_{12}^{S, k}, \mathbf{T}_{21}^{S, k}, \mathbf{T}_{22}^{S, k}$ are the $n^{X V} \times n^{X V}$ matrix elements of the $\mathbf{T}^{S, k}$ as evaluated from (10). Taking into account (9) and (10), the $2 n^{X V} \times 2 n^{X V}$ chain scattering matrix of a "branch" module, $\mathrm{T}^{k}$, is determined from $[3,40,41,71-73]$

$$
\mathbf{T}^{k}=\mathbf{T}^{T, k} \cdot \mathbf{T}^{S, k}=\left[\begin{array}{cc}
\mathbf{T}_{11}^{T, k} \mathbf{T}_{11}^{S, k}+\mathbf{T}_{12}^{T, k} \mathbf{T}_{21}^{S, k} & \mathbf{T}_{11}^{T, k} \mathbf{T}_{12}^{S, k}+\mathbf{T}_{12}^{T, k} \mathbf{T}_{22}^{S, k} \\
\mathbf{T}_{21}^{T, k} \mathbf{T}_{11}^{S, k}+\mathbf{T}_{22}^{T, k} \mathbf{T}_{21}^{S, k} & \mathbf{T}_{21}^{T, k} \mathbf{T}_{12}^{S, k}+\mathbf{T}_{22}^{T, k} \mathbf{T}_{22}^{S, k}
\end{array}\right]
$$

The last module of the overhead HV/BPL connection is the "load" module. Based on (9), the $2 n^{X V} \times 2 n^{X V}$ chain scattering matrix of the "load" module is given by

$$
\mathbf{T}^{N+1}=\left[\begin{array}{cc}
{\left[\mathbf{E}^{T, N+1}\right]^{-1}} & \mathbf{0}_{n^{X V} \times n^{X V}} \\
\mathbf{0}_{n^{X V} \times n^{X V}} & \mathbf{E}^{T, N+1}
\end{array}\right] .
$$

Through the multiplication rule of (11), the $2 n^{X V} \times 2 n^{X V}$ overall end-to-end chain scattering matrix is determined from

$$
\mathbf{T}^{\text {overall }}=\left[\begin{array}{cc}
\mathbf{T}_{11}^{\text {overall }} & \mathbf{T}_{12}^{\text {overall }} \\
\mathbf{T}_{21}^{\text {overall }} & \mathbf{T}_{22}^{\text {overall }}
\end{array}\right]=\prod_{k=1}^{N+1} \mathbf{T}^{k}
$$

where $\mathbf{T}_{11}^{\text {overall }}, \mathbf{T}_{12}^{\text {overall }}, \mathbf{T}_{21}^{\text {overall }}, \mathbf{T}_{22}^{\text {overall }}$ are the $n^{X V} \times n^{X V}$ matrix elements of the $\mathrm{T}^{\text {overall }}$ as evaluated from (13). Easily, the $2 n^{X V} \times 2 n^{X V}$ overall end-to-end scattering matrix is obtained from $[3,71,72]$

$S^{\text {overall }}$

$$
\begin{aligned}
& =\left[\begin{array}{ll}
\mathbf{S}_{11}^{\text {overall }} & \mathbf{S}_{12}^{\text {overall }} \\
\mathbf{S}_{21}^{\text {overall }} & \mathbf{S}_{22}^{\text {overall }}
\end{array}\right] \\
& =\left[\begin{array}{cc}
\mathbf{T}_{21}^{\text {overall }}\left[\mathbf{T}_{11}^{\text {overall }}\right]^{-1} & \mathbf{T}_{22}^{\text {overall }}-\mathbf{T}_{21}^{\text {overall }}\left[\mathbf{T}_{12}^{\text {overall }}\right]^{-1} \mathbf{T}_{11}^{\text {overall }} \\
{\left[\mathbf{T}_{11}^{\text {overall }}\right]^{-1}} & -\left[\mathbf{T}_{12}^{\text {overall }}\right]^{-1} \mathbf{T}_{11}^{\text {overall }}
\end{array}\right],
\end{aligned}
$$

where $\mathbf{S}_{11}^{\text {overall }}, \mathbf{S}_{12}^{\text {overall }}, \mathbf{S}_{21}^{\text {overall }}, \mathbf{S}_{22}^{\text {overall }}$ are the $n^{X V} \times n^{X V}$ elements of the $S^{\text {overall }}$ matrix as defined from (14).
Combining (4) and (14), the $n^{X V} \times n^{X V}$ EVD modal transfer function matrix is given by the $S_{21}^{\text {overall }}$ element of the $S^{\text {overall }}$ matrix; that is [3],

$$
\mathbf{H}^{X V, m}\{\cdot\}=\mathbf{S}_{21}^{\text {overall }}=\left[\mathbf{T}_{11}^{\text {overall }}\right]^{-1}
$$

Already proven in distribution BPL networks [3], the proposed TM2 method for overhead HV/BPL networks is extremely powerful since it is able to calculate EVD modal transfer functions associated with very elaborate networks including various types of overhead HV/BPL configurations, any type of interconnection at the branches, and any type of branch termination without assuming specific transmission assumptions as in SM and TM methods. Moreover, in contrast to SM and TM methods, the problem of mode mixture now can be fully investigated through the definition of the EVD modal XC transfer functions - as given in (15)-.

\section{Numerical Results and Discussion}

The simulations of various overhead HV/BPL transmission channels aim at investigating: (a) the validity and performance of the proposed TM2 method against other already theoretically and experimentally validated methods; (b) the broadband transmission characteristics of overhead HV/BPL channels and how these are affected by the overhead grid features, such as the type/topology of the overhead power grid and the multiplicity of branches; (c) the common $\mathrm{BPL} / \mathrm{PHY}$ handling potential between different types of overhead transmission power grid.

For the numerical computations, the $150 \mathrm{kV}$ singlecircuit, the $275 \mathrm{kV}$ double-circuit, and the $400 \mathrm{kV}$ doublecircuit overhead HV transmission line configurations, depicted in Figures 1(a), 1(b), and 1(c), respectively, have been considered. As previously mentioned, the modes supported by the overhead HV/BPL cable configurations may be examined separately. Thus, the following analysis is concentrated only on the EVD modal CC transfer functions.

The following discussion will focus on the transmission characteristics related to the $\mathrm{CM}^{X V}$ and to the $\mathrm{DM}^{X V}$ s of the overhead BPL systems, as well. Since, as demonstrated in [1, $2]$, the $\mathrm{DM}^{X V}$ s of the same overhead power grid type exhibit an almost identical spectral behavior, the transmission characteristics of only one $\mathrm{DM}^{X V}$ of each overhead power grid type, say that of $\mathrm{DM}_{4}^{X V}$, will be examined, hereafter.

The simple overhead topology of Figure 2(c), having $N$ branches, has been considered. In order to validate the accuracy of TM2 method and simplify the following analysis without affecting its generality, the branching cables are assumed identical to the transmission cables and the interconnections between the transmission and branch conductors are fully activated. With reference to Figure 2(c), the transmitting and the receiving ends are assumed matched to the characteristic impedance of the mode considered, whereas the branch terminations $\mathbf{Z}_{b k}, k=1,2, \ldots, N$, are assumed open circuit-that is, $\mathbf{K}_{b k}=\mathbf{I}_{n^{X V, b k}}, k=1,2, \ldots, N$, where $\mathbf{I}_{n^{X V, b k}}$ is the $n^{X V, b k} \times n^{X V, b k}$ identity matrix- [1$4,14,21-24,38,51-53,67]$. 
In accordance with [2], thousands of $\mathrm{km}$ of overhead $\mathrm{HV}$ lines are installed in more than 120 countries. These lines stretch from approximately $25 \mathrm{~km}$ to $190 \mathrm{~km}$ from the generation points before reaching any population centers. Shorter branches in the range of $10 \mathrm{~km}$ to $50 \mathrm{~km}$ are used in order to connect overhead HV transmission lines either between them or with $\mathrm{HV} / \mathrm{MV}$ substations $[1-4,10,14-$ $16,37,51-53,67,74-77]$.

With reference to Figure 2(c), four indicative overhead $\mathrm{HV}$ topologies concerning end-to-end connections of average lengths equal to $25 \mathrm{~km}$ are examined. These topologies, which are common for $150 \mathrm{kV}$ single-circuit, $275 \mathrm{kV}$ double-circuit, and $400 \mathrm{kV}$ double-circuit overhead HV/BPL systems, are $[4,10,14,51-53,67,76,77]$ as follows.

(1) A typical urban topology (urban case) with $N=3$ branches $\left(L_{1}=1.15 \mathrm{~km}, L_{2}=12.125 \mathrm{~km}, L_{3}=\right.$ $8.425 \mathrm{~km}, L_{4}=3.3 \mathrm{~km}, L_{b 1}=27.6 \mathrm{~km}, L_{b 2}=$ $\left.17.2 \mathrm{~km}, L_{b 3}=33.1 \mathrm{~km}\right)$.

(2) A typical suburban topology (suburban case) with $N=2$ branches $\left(L_{1}=9.025 \mathrm{~km}, L_{2}=12.75 \mathrm{~km}\right.$, $\left.L_{3}=3.225 \mathrm{~km}, L_{b 1}=46.8 \mathrm{~km}, L_{b 2}=13.4 \mathrm{~km}\right)$.

(3) A typical rural topology (rural case) with only $N=$ 1 branch $\left(L_{1}=3.75 \mathrm{~km}, L_{2}=21.25 \mathrm{~km}, L_{b 1}=\right.$ $21.1 \mathrm{~km})$.

(4) The "LOS" transmission along the average endto-end distance $L=L_{1}+\ldots+L_{N+1}=25 \mathrm{~km}$ when no branches are encountered. This topology corresponds to Line-of-Sight transmission in wireless channels.

To compare the proposed TM2 method with other established methods $[1,2,24,25,32,47,77,78]$ and simultaneously examine the end-to-end channel attenuation behavior for different overhead HV/BPL types and topologies, in Figures 3(a), 3(d), 3(g), and 3(j), the endto-end channel attenuation from $\mathrm{A}$ to $\mathrm{B}$ is plotted with respect to frequency for the propagation of $\mathrm{CM}^{150 \mathrm{kV}}$ for the aforementioned four indicative topologies, respectively, applying the proposed TM2 method, the TM method [1, $2,24,25]$, and the Multipath-Echo Based (MEB) method [32, 69, 77, 78]. In Figures 3(b), 3(e), 3(h), and 3(k), similar plots are drawn for the propagation of $\mathrm{CM}^{275 \mathrm{kV}}$ and in Figures 3(c), 3(f), 3(i), and 3(l) for $\mathrm{CM}^{400 \mathrm{kV}}$. In Figures 4(a)$4(\mathrm{l})$, similar curves are given for the propagation of $\mathrm{DM}_{4}^{X V}$ s.

From Figures 3(a)-3(1) and 4(a)-4(1) as it concerns the validity of TM2 method, it is observed that the three methods coincide in all the cases examined yielding excellent results regardless of the overhead power grid type, topology, and mode examined. Moreover, the TM2 method describes the spectral notches accurately either in depth or in spectral extent in any multipath environment. Thus, the TM2 method offers an accurate representation of the attenuation discontinuities caused by branching. It is also easily adapted to various network topologies resulting from various kinds of branches or HV/MV transformers. Due to the unique combination of generality and modular simplicity, the TM2 method will be adopted in the following simulations concerning the behavior of overhead HV/BPL transmission channels.

As for the end-to-end channel attenuation of overhead $\mathrm{HV} / \mathrm{BPL}$ channels, as it has already been investigated in [1$3,11,21-25,32,79-81]$, the spectral behavior of end-to-end channel attenuation depends drastically on the frequency, the mode considered, the physical properties of the cables used, the "LOS" distance, and the number and the length of the branches encountered along the end-to-end transmission path. Furthermore, as it has already been proposed in $[1,2,22-25,35,82]$ for other BPL channels, overhead $\mathrm{HV} / \mathrm{BPL}$ channels of transmission power grids may also be classified into three classes depending on the behavior of their spectral notches, namely, "LOS" channels, good channels, and bad channels. "LOS" case, rural case, and urban case A will represent "LOS", good, and bad channels, respectively, hereafter.

To demonstrate the effect of the branch length on the channel attenuation, in Figures 5(a) and 5(g), the endto-end channel attenuation from $\mathrm{A}$ to $\mathrm{B}$ is plotted versus frequency for good channel class case, Topology 1-same as good channel class case but with three times longer branches $\left(L_{1}=3.75 \mathrm{~km}, L_{2}=21.25 \mathrm{~km}, L_{b 1}=63.3 \mathrm{~km}\right)$, bad channel class case, Topology 2-same as bad channel class case but with three times longer branches $\left(L_{1}=1.15 \mathrm{~km}, L_{2}=\right.$ $12.125 \mathrm{~km}, L_{3}=8.425 \mathrm{~km}, L_{4}=3.3 \mathrm{~km}, L_{b 1}=82.8 \mathrm{~km}$, $L_{b 2}=51.6 \mathrm{~km}, L_{b 3}=99.3 \mathrm{~km}$ ), and "LOS" transmission case for the propagation of $\mathrm{CM}^{150 \mathrm{kV}}$ and $\mathrm{DM}_{4}^{150 \mathrm{kV}}$, respectively. In Figures 5(b), 5(c), 5(h), and 5(i), similar plots are drawn for the propagation of $\mathrm{CM}^{275 \mathrm{kV}}, \mathrm{CM}^{400 \mathrm{kV}}, \mathrm{DM}_{4}^{275 \mathrm{kV}}$, and $\mathrm{DM}_{4}^{400 \mathrm{kV}}$, respectively. Interesting results concerning the channel attenuation behavior of modes supported by the different overhead HV/BPL configurations may occur via their comparative analysis. Therefore, in Figures 5(d)-5(f), $5(j)-5(o)$, the absolute difference of the end-to-end channel attenuation from $\mathrm{A}$ to $\mathrm{B}$ is drawn versus frequency for the same topologies between: (i) $\mathrm{CM}^{150 \mathrm{kV}}$ and $\mathrm{CM}^{275 \mathrm{kV}}$; (ii) $\mathrm{CM}^{150 \mathrm{kV}}$ and $\mathrm{CM}^{400 \mathrm{kV}}$; (iii) $\mathrm{CM}^{275 \mathrm{kV}}$ and $\mathrm{CM}^{400 \mathrm{kV}}$; (iv) $\mathrm{DM}_{4}^{150 \mathrm{kV}}$ and $\mathrm{DM}_{4}^{275 \mathrm{kV}}$; (v) $\mathrm{DM}_{4}^{150 \mathrm{kV}}$ and $\mathrm{DM}_{4}^{400 \mathrm{kV} \text {; }}$ (vi) $\mathrm{DM}_{4}^{275 \mathrm{kV}}$ and $\mathrm{DM}_{4}^{400 \mathrm{kV}}$ (vii) $\mathrm{CM}^{150 \mathrm{kV}}$ and $\mathrm{DM}_{4}^{150 \mathrm{kV} \text {; }}$ (viii) $\mathrm{CM}^{275 \mathrm{kV}}$ and $\mathrm{DM}_{4}^{275 \mathrm{kV}}$; (ix) $\mathrm{CM}^{400 \mathrm{kV}}$ and $\mathrm{DM}_{4}^{400 \mathrm{kV}}$, respectively.

Observing Figures 3(a)-3(1), 4(a)-4(1), and 5(a)-5(o), it is evident that, due to reflections and multipath propagation caused by branches, spectral notches are observed in the channel attenuation, which are superimposed on the exponential "LOS" attenuation of each mode. However, due to the large branches of overhead HV/BPL topologies examined, the depth and the extent of the spectral notches are reduced in comparison with corresponding overhead LV/BPL and MV/BPL topologies [1-3, 21-25].

In addition, from Figures 5(d)-5(f) and 5(j)-5(l) the absolute difference of end-to-end channel attenuation between $\mathrm{CM}^{X V}$ and $\mathrm{DM}_{4}^{X V}$ of the same overhead HV/BPL grid type reveals the significant differences among the modes supported. However, due to the common bus-bar system topologies, the channel attenuation curves between $\mathrm{CM}^{X V} \mathrm{~s}$ and $\mathrm{DM}_{4}^{X V}$ s present similarities concerning the position and 


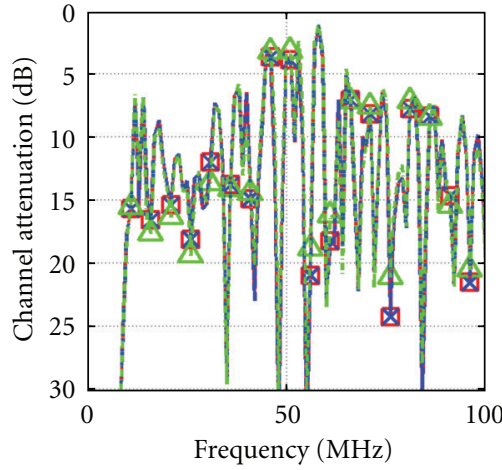

(a)

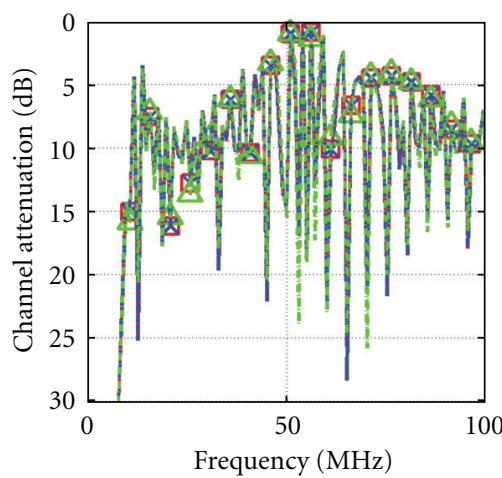

(d)

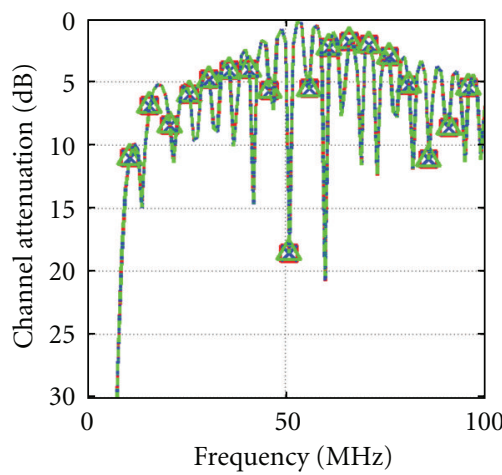

(g)

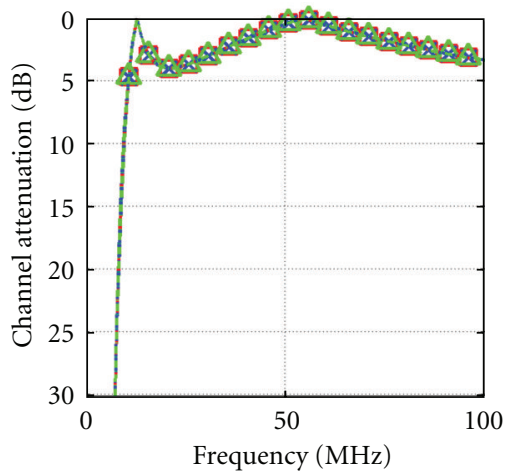

(j)

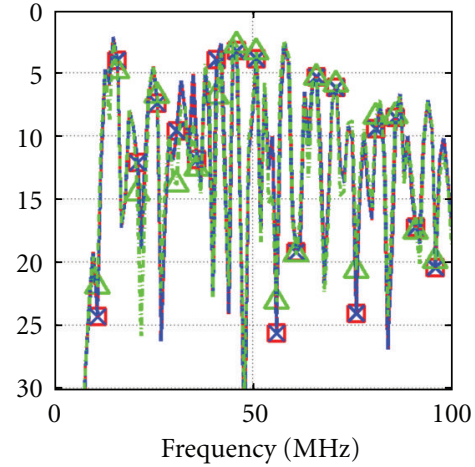

(b)

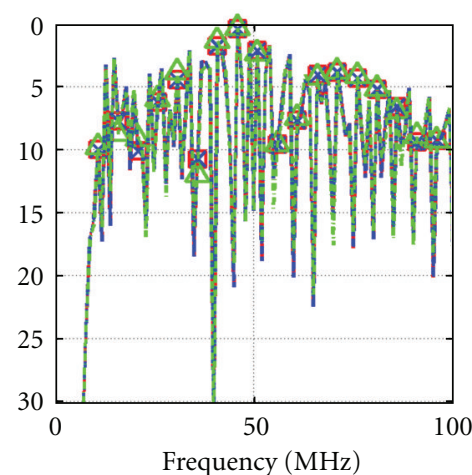

(e)

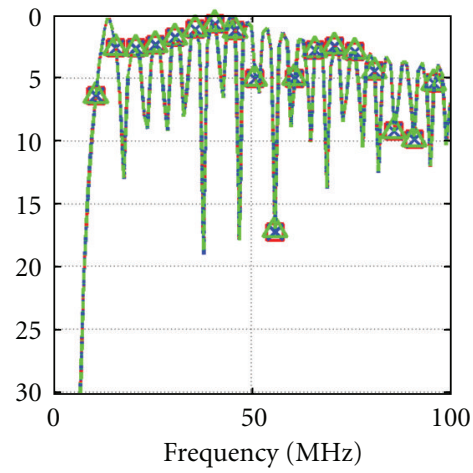

(h)

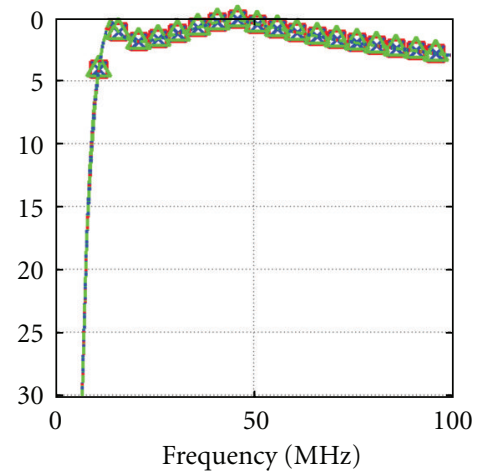

(k)

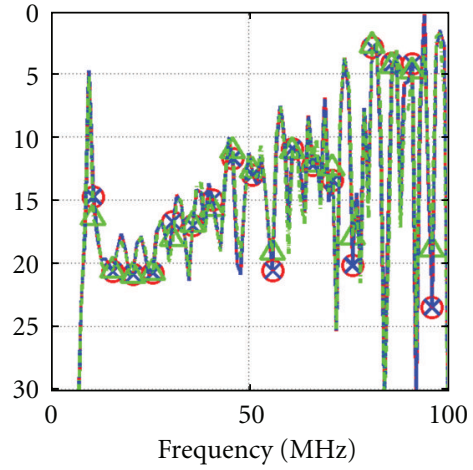

(c)

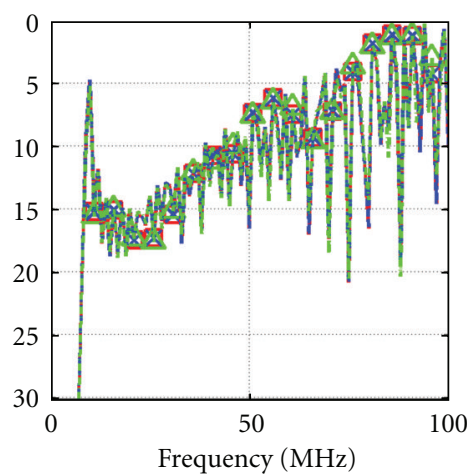

(f)

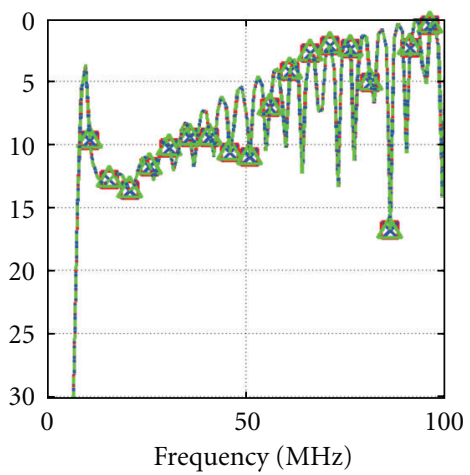

(i)

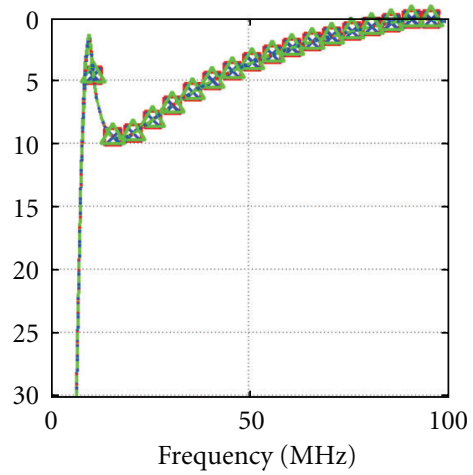

(l)

FIGURE 3: Application of the coinciding proposed TM2 method (red solid lined square), TM method (blue dashed line X), and MEB method (green dash-dotted triangle) to determine the end-to-end channel attenuation for the propagation of $\mathrm{CM}^{X V} \mathrm{~s}$ versus frequency for different overhead HV/BPL types and topologies (the subchannel frequency spacing is equal to $1 \mathrm{MHz}$ ). (a) Urban case $\mathrm{A} / 150 \mathrm{kV}$ single circuit. (b) Urban case A/275 kV double circuit. (c) Urban case A/400 kV double circuit. (d) Suburban case/150 kV single circuit. (e) Suburban case $/ 275 \mathrm{kV}$ double circuit. (f) Suburban case $/ 400 \mathrm{kV}$ double circuit. (g) Rural case $/ 150 \mathrm{kV}$ single circuit. (h) Rural case/275 kV double circuit. (i) Rural case $/ 400 \mathrm{kV}$ double circuit. (j) "LOS" case/150 kV single circuit. (k) "LOS” case/275 kV double circuit. (l) "LOS" case/400 kV double circuit. 


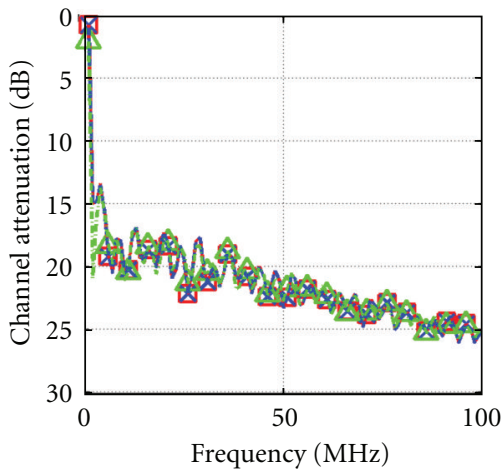

(a)

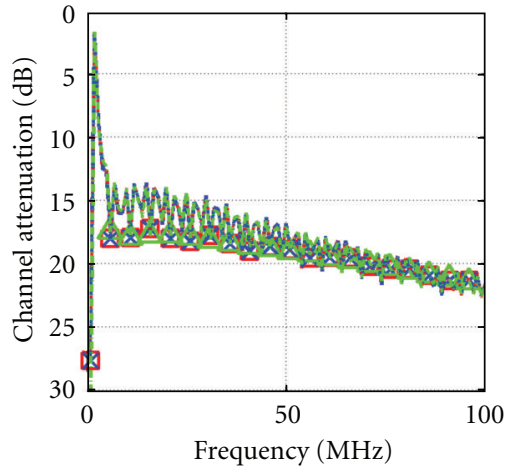

(d)

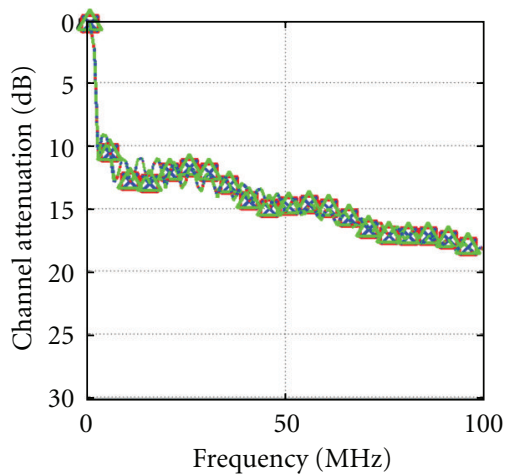

(g)

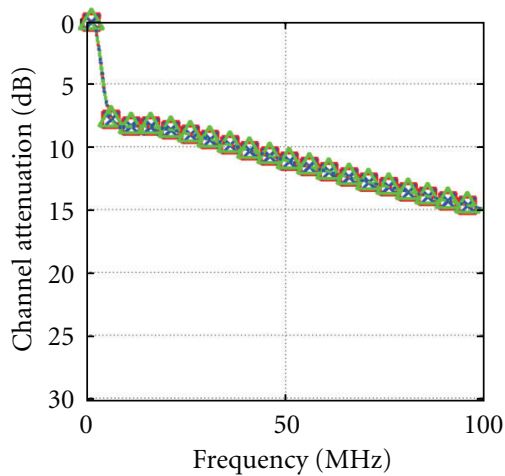

(j)

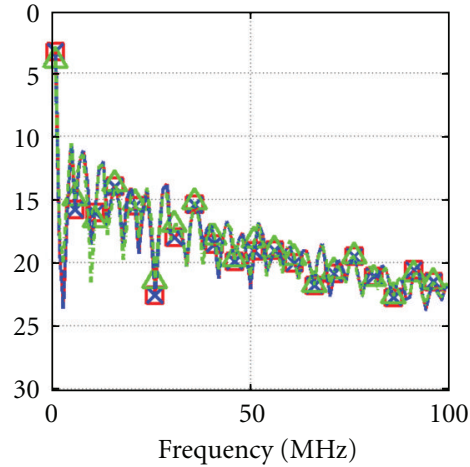

(b)

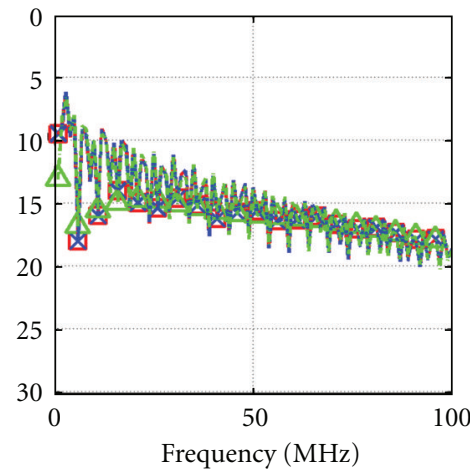

(e)

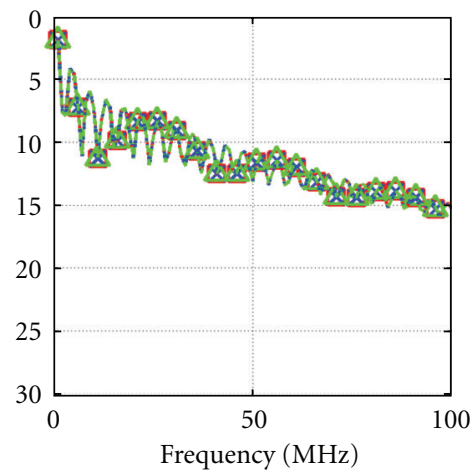

(h)

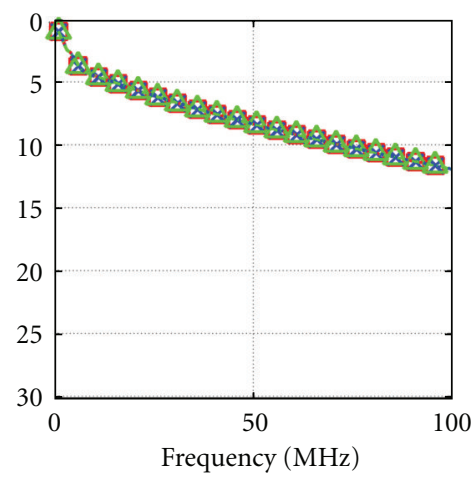

(k)

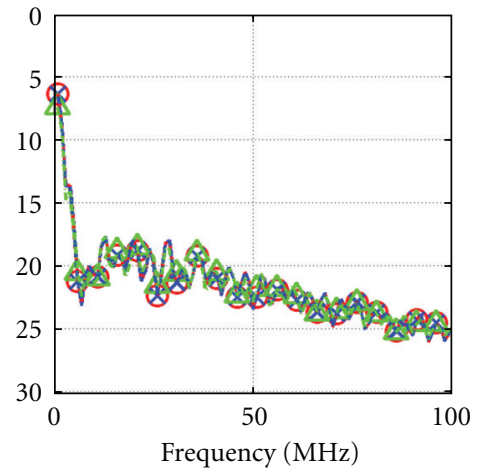

(c)

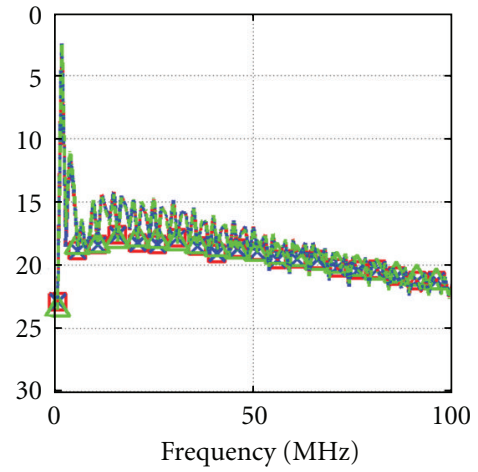

(f)

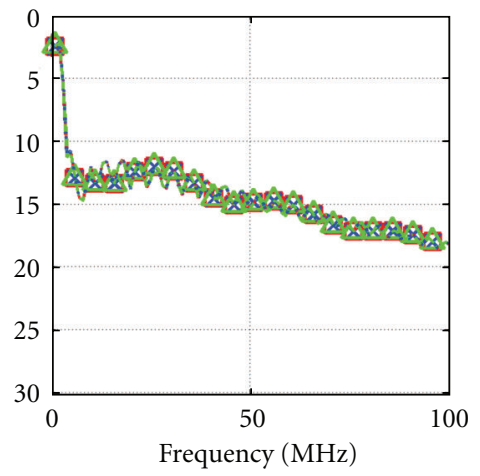

(i)

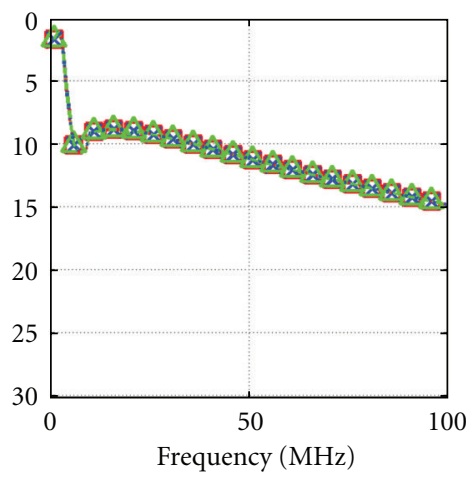

(l)

FIgURE 4: Same as in Figure 3, but for the propagation of $\mathrm{DM}_{4}^{X V}$ s. 


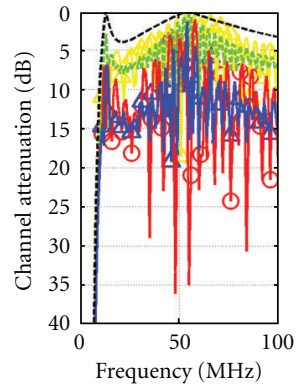

(a)

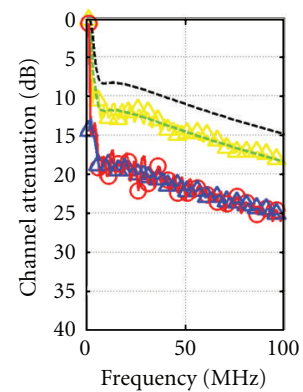

(g)

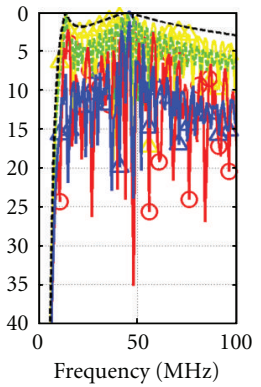

(b)

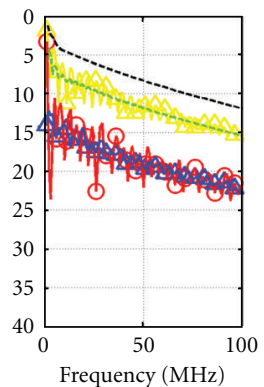

(h)

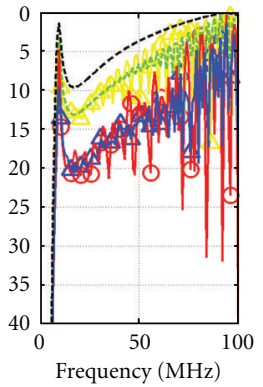

(c)

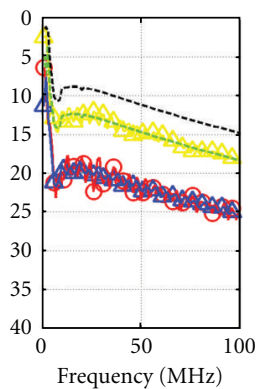

(i)

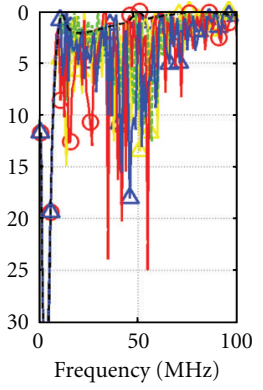

(d)

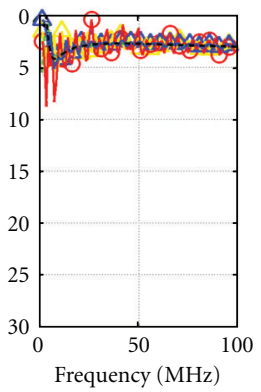

(j)

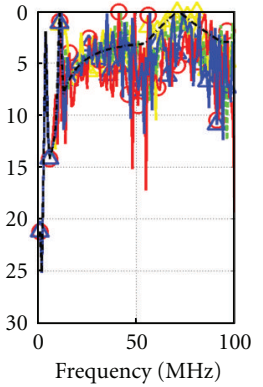

(e)

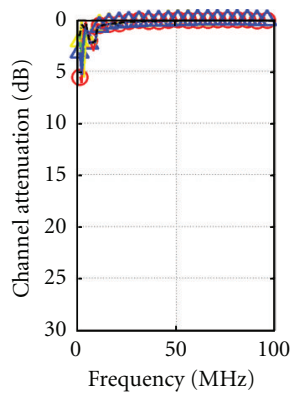

(k)

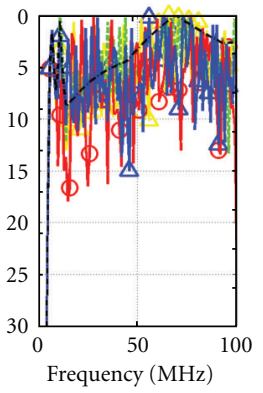

(f)

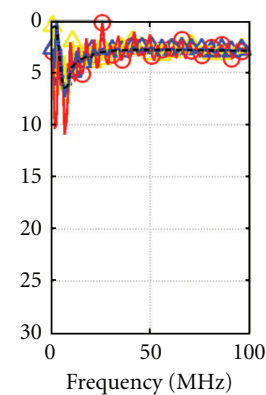

(1)

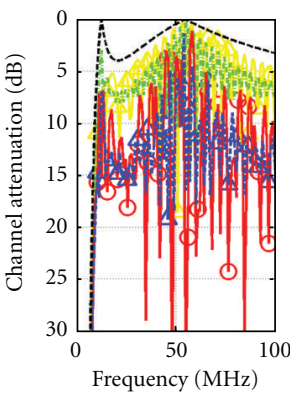

(m)

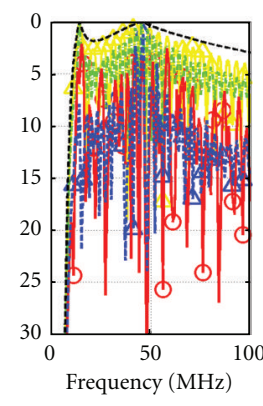

(n)

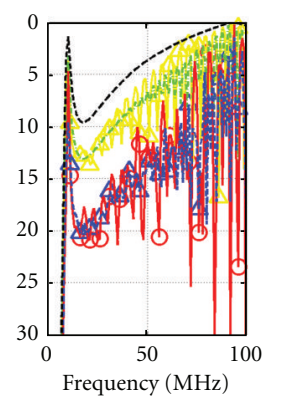

(o)

FIGURE 5: End-to-end channel attenuation versus frequency for good channel case (yellow solid lined triangle), Topology 1 (green dashed line), bad channel case (red solid lined circle), Topology 2 (blue dashed lined triangle), and "LOS" transmission case (black dashed line) (the subchannel frequency spacing is equal to $1 \mathrm{MHz}$ ). (a) $\mathrm{CM}^{150 \mathrm{kV}}$. (b) $\mathrm{CM}^{275 \mathrm{kV}}$. (c) $\mathrm{CM}^{400 \mathrm{kV}}$. (d) Absolute difference between $\mathrm{CM}^{150 \mathrm{kV}}$ and $\mathrm{CM}^{275 \mathrm{kV}}$. (e) Absolute difference between $\mathrm{CM}^{150 \mathrm{kV}}$ and $\mathrm{CM}^{400 \mathrm{kV}}$. (f) Absolute difference between $\mathrm{CM}^{275 \mathrm{kV}}$ and $\mathrm{CM}^{400 \mathrm{kV}}$. (g) $\mathrm{DM}_{4}^{150 \mathrm{kV}}$. (h) $\mathrm{DM}_{4}^{275 \mathrm{kV}}$. (i) $\mathrm{DM}_{4}^{400 \mathrm{kV}}$. (j) Absolute difference between $\mathrm{DM}_{4}^{150 \mathrm{kV}}$ and $\mathrm{DM}_{4}^{275 \mathrm{kV}}$. (k) Absolute difference between $\mathrm{DM}_{4}^{150 \mathrm{kV}}$ and $\mathrm{DM}_{4}^{400 \mathrm{kV}}$. (l) Absolute difference between $\mathrm{DM}_{4}^{275 \mathrm{kV}}$ and $\mathrm{DM}_{4}^{400 \mathrm{kV}}$. (m) Absolute difference between $\mathrm{CM}^{150 \mathrm{kV}}$ and $\mathrm{DM}_{4}^{150 \mathrm{kV}}$. (n) Absolute difference between $\mathrm{CM}^{275 \mathrm{kV}}$ and $\mathrm{DM}_{4}^{275 \mathrm{kV}}$. (o) Absolute difference between $\mathrm{CM}^{400 \mathrm{kV}}$ and $\mathrm{DM}_{4}^{400 \mathrm{kV}}$.

relative extent of spectral notches, confirming, thus, the strong correlation between the individual modal channels. In general, $\mathrm{DM}_{4}^{X V}$ s present higher channel attenuations than $\mathrm{CM}^{X V}$ s regardless of the overhead power grid type and topology. Anyway, as it is usually done to reduce the extent of results and simplify the analysis, an indicative picture of the transmission characteristics of the modes can be obtained studying the transmission characteristics of only one mode of each power grid type, say $\mathrm{DM}_{4}^{150 \mathrm{kV}}, \mathrm{DM}_{4}^{275 \mathrm{kV}}$, and $\mathrm{DM}_{4}^{400 \mathrm{kV}}$, hereafter, without affecting the generality of the analysis [13, 21-25].

As referred to in [1-3, 21-25], first, apart from causing spectral notches, the various branches also cause additional stepwise attenuation at each branch encountered along the end-to-end transmission path. Second, as the branch length increases, the stepwise attenuation at each branch tends to its corresponding matched branch termination one. This twofold effect of the branch length on the attenuation discontinuity at each branch is examined in Figures 6(a) and $6(\mathrm{~g})$, where the channel attenuation is plotted versus the distance from the transmitting end-see Figure 2(c), point $\mathrm{A}$-for the propagation of $\mathrm{DM}_{4}^{150 \mathrm{kV}}$ for good channel class case, Topology 1, Topology 3-same as good channel class case but with matched branch terminations, that is, $\mathbf{K}_{b k}=\mathbf{0}_{n^{X V, b k}}, k=1,2, \ldots, N$, where $\mathbf{0}_{n^{X V, b k}}$ is an $n^{X V, b k} \times$ $n^{X V, b k}$ matrix with zero elements, bad channel class case, Topology 2, Topology 4-same as bad channel class case but with matched branch terminations, and the "LOS" transmission case at $f=25 \mathrm{MHz}$ and $f=75 \mathrm{MHz}$, respectively. In Figures 6(b) and 6(h), similar plots are given 


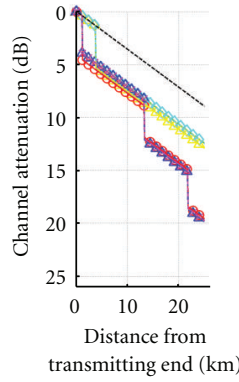

(a)

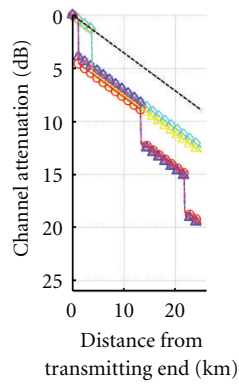

(g)

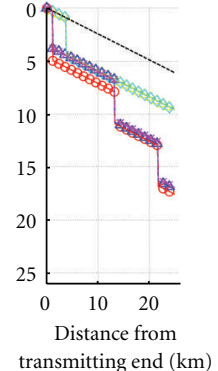

(b)

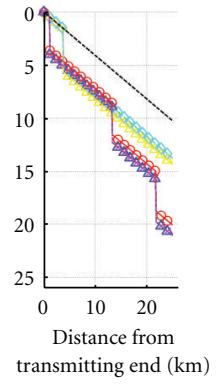

(h)

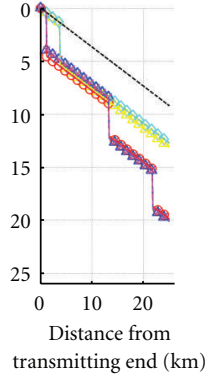

(c)

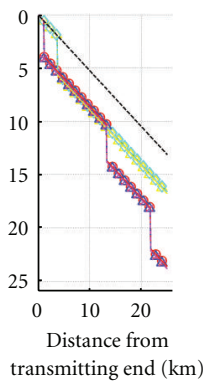

(i)

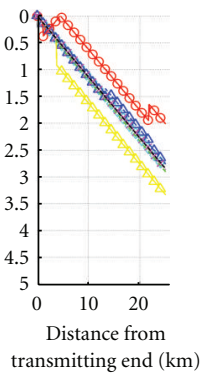

(d)

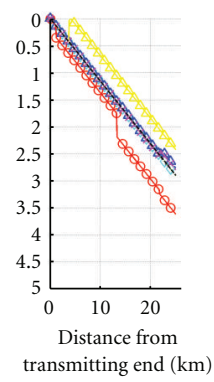

(j)

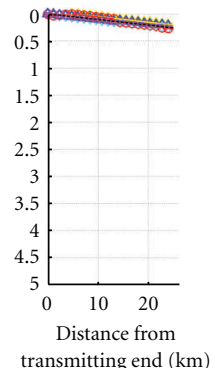

(e)

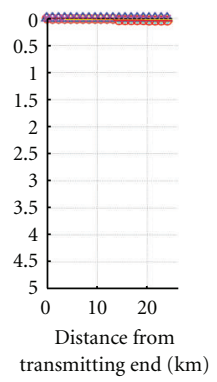

(k)

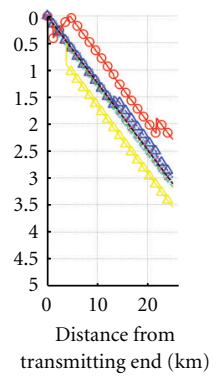

(f)

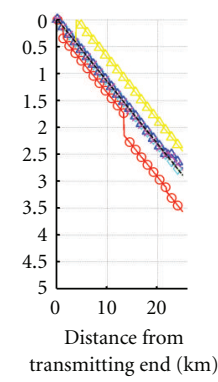

(1)

Figure 6: Channel attenuation versus the distance from the transmitting end-see Figure 2(c), point A-for good channel class case (yellow solid lined triangle), Topology 1 (green dashed line), Topology 3 (cyan solid lined diamond), bad channel case (red solid lined circle), Topology 2 (blue dashed lined triangle), Topology 4 (magenta dashed lined X), and "LOS" transmission case (black dash-dotted line) (the distance span is equal to $100 \mathrm{~m}$ ). (a) $\mathrm{DM}_{4}^{150 \mathrm{kV}}$ at $f=25 \mathrm{MHz}$. (b) $\mathrm{DM}_{4}^{275 \mathrm{kV}}$ at $f=25 \mathrm{MHz}$. (c) $\mathrm{DM}_{4}^{400 \mathrm{kV}}$ at $f=25 \mathrm{MHz}$. (d) $\mathrm{Absolute}$ difference between $\mathrm{DM}_{4}^{150 \mathrm{kV}}$ and $\mathrm{DM}_{4}^{275 \mathrm{kV}}$ at $f=25 \mathrm{MHz}$. (e) Absolute difference between $\mathrm{DM}_{4}^{150 \mathrm{kV}}$ and $\mathrm{DM}_{4}^{400 \mathrm{kV}}$ at $f=25 \mathrm{MHz}$. (f) Absolute difference between $\mathrm{DM}_{4}^{275 \mathrm{kV}}$ and $\mathrm{DM}_{4}^{400 \mathrm{kV}}$ at $f=25 \mathrm{MHz}$. (g) $\mathrm{DM}_{4}^{150 \mathrm{kV}}$ at $f=75 \mathrm{MHz}$. (h) $\mathrm{DM}_{4}^{275 \mathrm{kV}}$ at $f=75 \mathrm{MHz}$. (i) $\mathrm{DM}_{4}^{400 \mathrm{kV}}$ at $f=75 \mathrm{MHz}$. (j) Absolute difference between $\mathrm{DM}_{4}^{150 \mathrm{kV}}$ and $\mathrm{DM}_{4}^{275 \mathrm{kV}}$ at $f=75 \mathrm{MHz}$. (k) Absolute difference between $\mathrm{DM}_{4}^{150 \mathrm{kV}}$ and $\mathrm{DM}_{4}^{400 \mathrm{kV}}$ at $f=75 \mathrm{MHz}$. (l) Absolute difference between $\mathrm{DM}_{4}^{275 \mathrm{kV}}$ and $\mathrm{DM}_{4}^{400 \mathrm{kV}}$ at $f=75 \mathrm{MHz}$.

for the propagation of $\mathrm{DM}_{4}^{275 \mathrm{kV}}$ and in Figures 6(c) and 6(i) for $\mathrm{DM}_{4}^{400 \mathrm{kV}}$. Interesting remarks may be pointed out studying the differences among the modes supported by each overhead HV configuration. Thus, in Figures 6(d), 6(e), and $6(\mathrm{f})$, the absolute difference of the channel attenuation between, (i) $\mathrm{DM}_{4}^{150 \mathrm{kV}}$ and $\mathrm{DM}_{4}^{275 \mathrm{kV}}$, (ii) $\mathrm{DM}_{4}^{150 \mathrm{kV}}$ and $\mathrm{DM}_{4}^{400 \mathrm{kV}}$, and (iii) $\mathrm{DM}_{4}^{275 \mathrm{kV}}$ and $\mathrm{DM}_{4}^{400 \mathrm{kV}}$; respectively, is also drawn with respect to the distance from the transmitting end for the same topologies at $f=25 \mathrm{MHz}$. In Figures 6(j)6(1), similar plots are given at $f=75 \mathrm{MHz}$.

From Figures 6(a)-6(l), it is demonstrated that the attenuation discontinuity at each branch depends primarily on the frequency rather than overhead transmission power grid type and branch length. Indeed, from Figures 6(a)6(c) and 6(g)-6(i), even though good channel class case, Topology 1, and Topology 3 present significant topological differences, the strong role of the branch length defines their almost identical spectral behavior. Same results occur among bad channel class case, Topology 2, and Topology 4. However, the influence of branch length is an intertopological factor; from Figures 6(d)-6(f) and 6(j)-6(l), it is evident that the channel attenuation differences are lower than $3 \mathrm{~dB}$ in the majority of the cases for given overhead power grid topology regardless of the overhead power grid type.

The above results are attributed to the inherent spectral features of overhead HV transmission; as the branch lengths increase, which are already extremely longer in comparison with the respective branch lengths of overhead LV/BPL and MV/BPL grids, the spectral behavior of overhead HV/BPL networks tends to converge to that of overhead ones with matched branch terminations-see Figures $5(\mathrm{a})-5(\mathrm{c}), 5(\mathrm{~g})-$ $5(\mathrm{i}), 6(\mathrm{a})-6(\mathrm{c})$, and $6(\mathrm{~g})-6(\mathrm{i})$ - . In the case of matched branch terminations, relating (7)-(11) with (8) from [24], $\mathbf{T}^{k}(f)$ mainly depends on $\mathbf{E}^{T, k}(f)$ rather than $\mathbf{K}_{b k}$ which exhibits static behavior in respect with frequency, say $\mathbf{K}_{b k}=$ $\mathbf{K}_{b k}(f)=\mathbf{0}_{n^{x V}, b k}, k=1,2, \ldots, N$. The disclosure of this quasi-static spectral behavior of overhead HV/BPL systems is significant since the main problem of overhead transmission and distribution power grids is their intense time- and frequency-selective channel attenuation variation due to various factors, such as sudden new loads, blackouts, synchronization problems, renewable energy source production, power exchange issues, connection/disconnection of large industrial/sensitive electrical loads, and other cascading failures $[1-3,21-31]$. This rule of thumb allows better broadband monitoring and management of overhead HV transmission power grids in an interactive SG network bypassing an elaborate broadband analysis of the overhead transmission network.

Except for the single braches at given junction, similar to overhead LV/BPL and MV/BPL networks, a typical urban overhead HV topology can support 1-5 HV/MV 


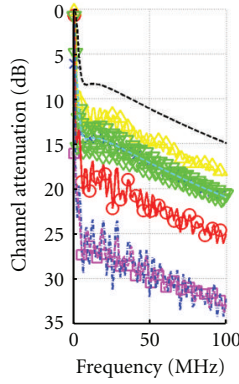

(a)

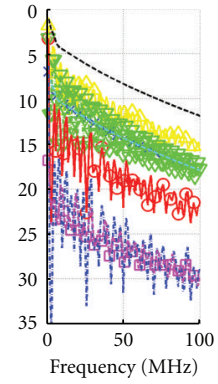

(b)

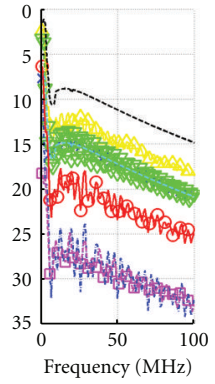

(c)

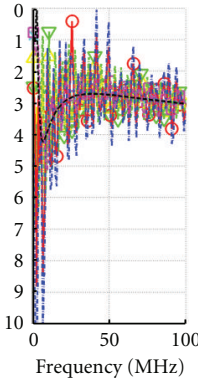

(d)

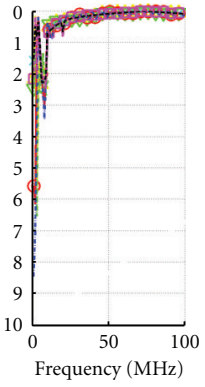

(e)

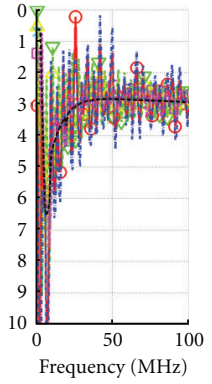

(f)

FIGURE 7: End-to-end channel attenuation versus frequency for good channel class case (yellow solid lined triangle), Topology 5 (green dashed lined reversed triangle), Topology 6 (cyan dashed lined X), bad channel class case (red solid lined circle), Topology 7 (blue dashdotted line), Topology 8 (magenta dashed lined square), and "LOS" transmission case (black dashed line) (the subchannel frequency spacing is equal to $1 \mathrm{MHz}$ ). (a) $\mathrm{DM}_{4}^{150 \mathrm{kV}}$. (b) $\mathrm{DM}_{4}^{275 \mathrm{kV}}$. (c) $\mathrm{DM}_{4}^{400 \mathrm{kV}}$. (d) Absolute difference between $\mathrm{DM}_{4}^{150 \mathrm{kV}}$ and $\mathrm{DM}_{4}^{275 \mathrm{kV}}$. (e) $\mathrm{Absolute}$ difference between $\mathrm{DM}_{4}^{150 \mathrm{kV}}$ and $\mathrm{DM}_{4}^{400 \mathrm{kV}}$. (f) Absolute difference between $\mathrm{DM}_{4}^{275 \mathrm{kV}}$ and $\mathrm{DM}_{4}^{400 \mathrm{kV}}$.

transformers. These overhead topologies are mainly of radial configuration either with a single branch or with multiple branches at the same junction $[3,9,10,15,16,23,28,43,46$, $47,78]$.

To demonstrate the effect of multiple branches at given junction on the channel attenuation, in Figure 7(a), the endto-end channel attenuation from $\mathrm{A}$ to $\mathrm{B}$ for the propagation of $\mathrm{DM}_{4}^{150 \mathrm{kV}}$ is plotted versus frequency for good channel class case, Topology 5-same as good channel class case but with two times more, same branches at each junction $\left(L_{1}=3.75 \mathrm{~km}, L_{2}=0 \mathrm{~km}, L_{3}=21.25 \mathrm{~km}, L_{b 1}=L_{b 2}=\right.$ $21.1 \mathrm{~km}$ ), Topology 6-same as Topology 5 but with matched branch terminations, bad channel class case, Topology 7same as bad channel class case but with two times more, same branches at each junction $\left(L_{1}=1.15 \mathrm{~km}, L_{2}=0 \mathrm{~km}\right.$, $L_{3}=12.125 \mathrm{~km}, L_{4}=0 \mathrm{~km}, L_{5}=8.425 \mathrm{~km}, L_{6}=0 \mathrm{~km}$, $L_{7}=3.3 \mathrm{~km}, L_{b 1}=L_{b 2}=27.6 \mathrm{~km}, L_{b 3}=L_{b 4}=17.2 \mathrm{~km}$, $L_{b 5}=L_{b 6}=33.1 \mathrm{~km}$ ), Topology 8-same as Topology 7 but with matched branch terminations, and "LOS" transmission case. In Figures 7(b) and 7(c), similar plots are given for the propagation of $\mathrm{DM}_{4}^{275 \mathrm{kV}}$ and $\mathrm{DM}_{4}^{400 \mathrm{kV}}$, respectively. In order to examine the differences among $\mathrm{DM}_{4}^{X V}$ s for these overhead HV topologies, in Figures 7(d), 7(e), and 7(f), the absolute difference of the channel attenuation between, (i) $\mathrm{DM}_{4}^{150 \mathrm{kV}}$ and $\mathrm{DM}_{4}^{275 \mathrm{kV}}$, (ii) $\mathrm{DM}_{4}^{150 \mathrm{kV}}$ and $\mathrm{DM}_{4}^{400 \mathrm{kV}}$, and (iii) $\mathrm{DM}_{4}^{275 \mathrm{kV}}$ and $\mathrm{DM}_{4}^{400 \mathrm{kV}}$, respectively, is also drawn with respect to frequency for the same topologies. Note that the cases examined correspond to rather special and aggravated transmission cases. However, useful remarks may be pointed out regarding multiple-branch junctions and corresponding matched branch termination networks.

From Figures $7(\mathrm{a})-7(\mathrm{c})$, it is observed that the multiple branches at each junction cause additional stepwise attenuation deteriorating the existing stepwise attenuation due to single branches. However, the effect of the multiple branches may be cumulatively explained studying the attenuation discontinuity at the each junction.

More analytically, in Figures 8(a) and 8(g), the channel attenuation is plotted versus the distance from the transmitting end-see Figure 2(c), point A—for the propagation of
$\mathrm{DM}_{4}^{150 \mathrm{kV}}$ for good channel class case, Topology 5, Topology 6 , bad channel class case, Topology 7, Topology 8 , and the "LOS" transmission case at $f=25 \mathrm{MHz}$ and $f=75 \mathrm{MHz}$, respectively. In Figures 8(b) and 8(h), similar plots are given for the propagation of $\mathrm{DM}_{4}^{275 \mathrm{kV}}$ and in Figures 6(c) and 6(i) for $\mathrm{DM}_{4}^{400 \mathrm{kV}}$. Interesting convergence results will be reported to the comparative modal analysis subsection that follows by examining the behavior of $\mathrm{DM}_{4}^{X V}$ s for different overhead HV topologies; in Figures 8(d), 8(e), and 8(f), the absolute difference of the channel attenuation between (i) $\mathrm{DM}_{4}^{150 \mathrm{kV}}$ and $\mathrm{DM}_{4}^{275 \mathrm{kV}}$, (ii) $\mathrm{DM}_{4}^{150 \mathrm{kV}}$ and $\mathrm{DM}_{4}^{400 \mathrm{kV}}$, and (iii) $\mathrm{DM}_{4}^{275 \mathrm{kV}}$ and $\mathrm{DM}_{4}^{400 \mathrm{kV}}$, respectively, is also drawn with respect to the distance from the transmitting end for the same topologies at $f=25 \mathrm{MHz}$. In Figures $8(\mathrm{j})-8(\mathrm{l})$, similar plots are given at $f=75 \mathrm{MHz}$.

Figures $7(\mathrm{a})-7(\mathrm{c}), 8(\mathrm{a})-8(\mathrm{c})$, and $8(\mathrm{~g})-8(\mathrm{i})$ clearly show the destructive effect of branch presence; the attenuation exhibited by overhead HV/BPL channels when single/multiple branches are added across the BPL signal transmission is dramatically worse than the attenuation of overhead "LOS" ones. Multiple branches critically aggravate the channel performance. Therefore, during the implementation of overhead HV/BPL networks in urban and suburban environments, denser overhead HV/BPL networks are preferable.

Moreover, the quasi-static spectral behavior of overhead $\mathrm{HV} / \mathrm{BPL}$ systems is also validated in overhead transmission power grids with multiple branches per junction. Observing the above figures, even though Topology 5 and Topology 6 present significant differences regarding their branch terminations, their channel attenuation and attenuation discontinuity are very close in all the cases examined. Same results occur between Topology 7 and Topology 8 permitting to generalize the quasi-static spectral behavior consideration in every overhead HV/BPL grid considered.

5.1. Comparative Modal Analysis and Discussion. Concluding the above comparative modal analysis concerning the behavior of overhead HV/BPL grid and its supported overhead 


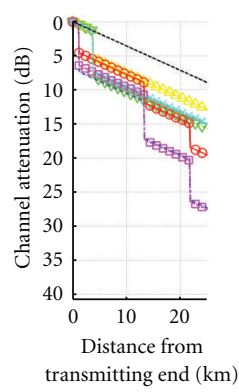

(a)

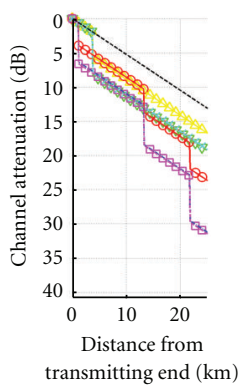

(g)

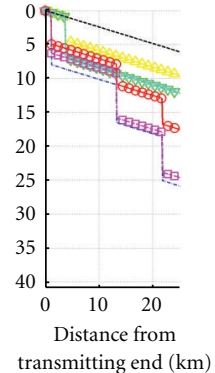

(b)

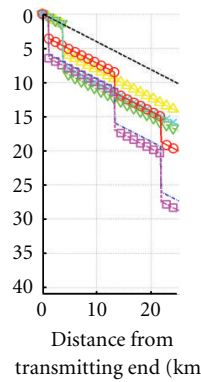

(h)

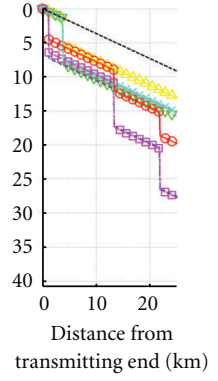

(c)

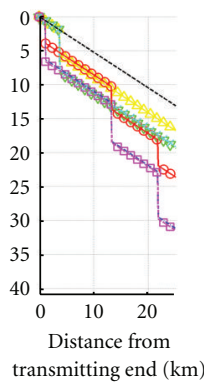

(i)

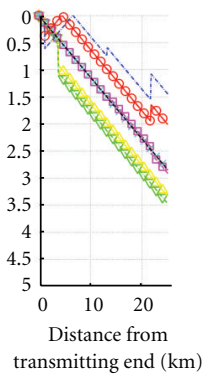

(d)

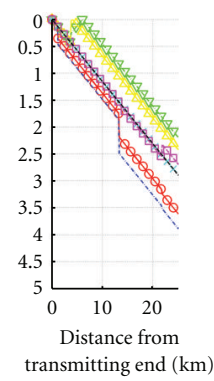

(j)

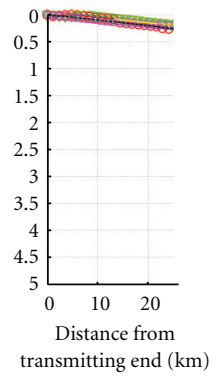

(e)

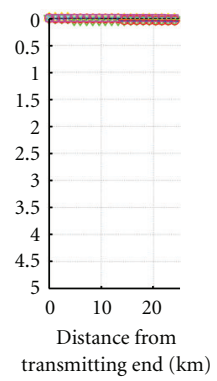

(k)

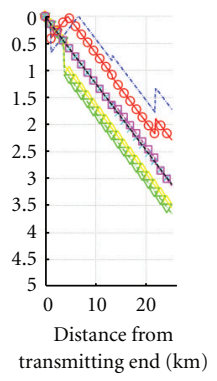

(f)

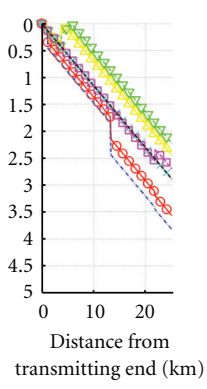

(1)

FIGURE 8: Channel attenuation versus the distance from the transmitting end-see Figure 2(c), point A—for good channel class case (yellow solid lined triangle), Topology 5 (green dashed lined reversed triangle), Topology 6 (cyan dashed lined X), bad channel class case (red solid lined circle), Topology 7 (blue dash-dotted line), Topology 8 (magenta dashed lined square), and "LOS" transmission case (black dashed line) (the distancespan is equal to $100 \mathrm{~m}$ ). (a) $\mathrm{DM}_{4}^{150 \mathrm{kV}}$ at $f=25 \mathrm{MHz}$. (b) $\mathrm{DM}_{4}^{275 \mathrm{kV}}$ at $f=25 \mathrm{MHz}$. (c) $\mathrm{DM}_{4}^{400 \mathrm{kV}}$ at $f=25 \mathrm{MHz}$. (d) Absolute difference between $\mathrm{DM}_{4}^{150 \mathrm{kV}}$ and $\mathrm{DM}_{4}^{275 \mathrm{kV}}$ at $f=25 \mathrm{MHz}$. (e) Absolute difference between $\mathrm{DM}_{4}^{150 \mathrm{kV}}$ and $\mathrm{DM}_{4}^{400 \mathrm{kV}}$ at $f=25 \mathrm{MHz}$. (f) Absolute difference between $\mathrm{DM}_{4}^{275 \mathrm{kV}}$ and $\mathrm{DM}_{4}^{400 \mathrm{kV}}$ at $f=25 \mathrm{MHz}$. (g) $\mathrm{DM}_{4}^{150 \mathrm{kV}}$ at $f=75 \mathrm{MHz}$. (h) $\mathrm{DM}_{4}^{275 \mathrm{kV}}$ at $f=75 \mathrm{MHz}$. (i) $\mathrm{DM}_{4}^{400 \mathrm{kV}}$ at $f=75 \mathrm{MHz}$. (j) Absolute difference between $\mathrm{DM}_{4}^{150 \mathrm{kV}}$ and $\mathrm{DM}_{4}^{275 \mathrm{kV}}$ at $f=75 \mathrm{MHz}$. (k) Absolute difference between $\mathrm{DM}_{4}^{150 \mathrm{kV}}$ and $\mathrm{DM}_{4}^{400 \mathrm{kV}}$ at $f=75 \mathrm{MHz}$. (l) Absolute difference between $\mathrm{DM}_{4}^{275 \mathrm{kV}}$ and $\mathrm{DM}_{4}^{400 \mathrm{kV}}$ at $f=75 \mathrm{MHz}$.

MTL configurations see Figures 1(a)-1(c), several interesting conclusions may be deduced.

(i) The $\mathrm{CM}^{150 \mathrm{kV}}, \mathrm{CM}^{275 \mathrm{kV}}$, and $\mathrm{CM}^{400 \mathrm{kV}}$ exhibit very close end-to-end channel attenuations for a great number of indicative overhead BPL topologies-see Figures 3(a)-3(l) and Figures 5(a)-5(c)- . Hence, the transmission characteristics of only one $\mathrm{CM}^{X V}-$ either $\mathrm{CM}^{150 \mathrm{kV}}$ or $\mathrm{CM}^{275 \mathrm{kV}}$ or $\mathrm{CM}^{400 \mathrm{kV}}$ —may be examined for all overhead HV/BPL systems with satisfactory accuracy as shown through their comparative analysis—see Figures 5(d) -5(f) - .

(ii) As to the $\mathrm{DM}^{X V} \mathrm{~s}$, the $\mathrm{DM}^{150 \mathrm{kV}} \mathrm{s}, \mathrm{DM}^{275 \mathrm{kV}} \mathrm{s}$, and $\mathrm{DM}^{400 \mathrm{kV}}$ s exhibit an almost identical spectral behavior regarding their attenuation coefficients and phase delays as verified in $[1-3,21-25]$. Due to this spectral behavior, only one of the supported modes per each overhead HV/BPL transmission grid, say $\mathrm{DM}_{4}^{X V}$ s, may be examined offering a representative image for all the other $\mathrm{DM}^{X V}$ s supported. Moreover, from Figures 4(a)-4(l), 5(g)-5(i), 6(a)-6(l), 7(a)-7(f), and $8(a)-8(1)$ it is validated the very close either end-toend channel attenuation or attenuation discontinuity among $\mathrm{DM}_{4}^{150 \mathrm{kV}}, \mathrm{DM}_{4}^{275 \mathrm{kV}}$, and $\mathrm{DM}_{4}^{400 \mathrm{kV}}$ for a great number of overhead HV/BPL grid topologies. More specifically, through the comparative analysis of $\mathrm{DM}_{4}^{X V}$ s-see Figures 5(j)-5(l), 6(d)-6(f), and 6(j)$6(1)-$, it is verified that the transmission characteristics of only one of $\mathrm{DM}_{4}^{X V}$ s-only one of either $\mathrm{DM}_{4}^{150 \mathrm{kV}}$ or $\mathrm{DM}_{4}^{275 \mathrm{kV}}$ or $\mathrm{DM}_{4}^{400 \mathrm{kV}}$ — may be examined giving results with excellent accuracy for the entire overhead HV/BPL transmission grid. The remarkable convergence of this approximation is maintained also to multibranch junction networks as illustrated in Figures 7(d)-7(f), 8(d)-8(f), and 8(j)$8(1)$.

(iii) The common PHY framework of overhead HV/BPL configurations extends the efforts of $[1,2,25]$ towards overhead transmission power system integration. As demonstrated in Figures 5(j)-5(l), only the transmission characteristics of one of either $\mathrm{CM}^{X V}$ or $\mathrm{DM}_{4}^{X V}$ may be assumed offering common conclusions for all overhead HV/BPL configurations examined with satisfactory accuracy. This consideration of only one mode, say $\mathrm{DM}_{4}^{150 \mathrm{kV}}$, for all overhead HV/BPL transmission grid defines the final step of the proposed common handling PHY approach. 
Synopsizing the above analysis, several useful conclusions and discussion issues are raised, namely, the following.

(1) Though determined for $25 \mathrm{~km}$ long HV connections - compared to the significantly shorter connections of LV and MV cases $[4,10,11,21-24,26,27$, $36,38,39,81]$, BPL transmission via the overhead HV grid exhibits low-loss features, impressive flatfading characteristics, and low multipath dispersion rendering, thus, HV/BPL transmission as a fiberoptic transmission alternative solution $[1,2]$.

(2) The common PHY framework gives a representative image of the overall end-to-end channel attenuation and permits the study of the spectral behavior of overhead HV/BPL networks by examining only one mode supported by the various overhead MTL configurations that constitute the overhead $\mathrm{HV}$ transmission power grid. This approach offers (i) a useful tool with low complexity towards BPL/SG systems coexistence and unified BPL network design and (ii) necessary rules of thumb in order to bypass the complicated EVD modal analyses.

(3) Based on the well-established hybrid model of [1$3,21-25]$ and the generic multidimensional network analysis tool presented in $[3,36,42,43]$, an extremely powerful multidimensional chain scattering matrix method-TM2 method-is presented. Already proven in distribution BPL networks [3], TM2 method is now able to calculate EVD modal transfer functions associated with very elaborate networks including various types of overhead HV/BPL configurations, any type of interconnection at the branches, and any type of branch termination without imposing special simplifying transmission assumptions. In addition, TM2 method provides significant guidance regarding mode mixture issues via the definition of EVD modal XC transfer functions which describe the crosstalk couplings among the modes supported. Finally, TM2 method is well validated against other theoretically and experimentally proven models, such as MEB and TM methods.

(4) As it has already been proposed in $[4-8,69,83,84]$ for other LV/BPL, MV/BPL, and HV/BPL channels, overhead HV/BPL channels of transmission power grids may be classified into three classes depending on the behavior of their spectral notches: "LOS" channels, good channels, and bad channels.

(5) As the branches become longer, the spectral behavior of the overhead BPL networks tends to converge to the spectral behavior of equivalent circuits which consist of matched branch terminations. This remark is also validated in the multi-branch junction overhead HV/BPL networks. This rule of thumb may help towards better broadband monitoring and management of HV transmission grids in the upcoming SG networks.

\section{Conclusions}

This paper has focused on a new exact multidimensional chain scattering matrix method (TM2 method) appropriate for overhead HV/BPL networks and the unified handling potential of overhead HV/BPL configurations through the common PHY framework extension. The applied TM2 method combined with the extended common PHY framework offers a valuable tool towards the integrated BPL transmission network design.

Also reported in other LV/BPL and MV/BPL networks, the TM2 method has revealed that the broadband transmission capability of overhead HV/BPL networks depends on the frequency, the physical properties of the overhead MTL configuration used, the "LOS" distance, and the number, the length, the terminations, and the multiplicity of the branches along the end-to-end BPL signal propagation. Overhead HV/BPL channels have been classified into three classes: "LOS" channels, good channels, and bad channels. Managing the common PHY framework and exploiting the inherent long-branch structure of overhead HV grid, a simple quasi-static approach suitable for the channel modeling of overhead HV/BPL transmission grid has been proposed.

Finally, the results validate the low-loss nature of overhead HV/BPL grid over a $25 \mathrm{~km}$ repeater span well beyond $100 \mathrm{MHz}$ regardless of the overhead $\mathrm{HV}$ power grid type and topology, hence, rendering it as a promising broadband SG partner.

\section{References}

[1] A. G. Lazaropoulos, "Broadband transmission characteristics of overhead high-voltage power line communication channels," Progress in Electromagnetics Research B, vol. 36, pp. 373398, 2012.

[2] A. G. Lazaropoulos, "Broadband transmission and statistical performance properties of overhead high-voltage transmission networks," Journal of Computer Networks and Commun, vol. 2012, Article ID 875632, 16 pages, 2012.

[3] A. G. Lazaropoulos, "Towards modal integration of overhead and underground low-voltage and medium-voltage power line communication channels in the smart grid landscape: model expansion, broadband signal transmission characteristics, and statistical performance metrics (invited paper)," ISRN Signal Processing, vol. 2012, Article ID 121628, 17 pages, 2012.

[4] OPERA1, "D44: Report presenting the architecture of plc system, the electricity network topologies, the operating modes and the equipment over which PLC access system will be installed," IST Integrated Project 507667, 2005.

[5] N. Pavlidou, A. J. Han Vinck, J. Yazdani, and B. Honary, "Power line communications: state of the art and future trends," IEEE Communications Magazine, vol. 41, no. 4, pp. 34-40, 2003.

[6] G. N. S. Prasanna, A. Lakshmi, S. Sumanth, V. Simha, J. Bapat, and G. Koomullil, "Data communication over the smart grid," in Proceedings of the IEEE International Symposium on Power Line Communications and its Applications (ISPLC '09), pp. 273-279, Dresden, Germany, April 2009. 
[7] NATO, "HF Interference, Procedures and Tools (Interférences HF, procédures et outils) Final Report of NATO RTO Information Systems Technology," Tech. Rep. RTO-TR-ISTR-050, 2007.

[8] M. P. Anastasopoulos, A. C. Voulkidis, A. V. Vasilakos, and P. G. Cottis, "A secure network management protocol for SmartGrid BPL networks: design, implementation and experimental results," Computer Communications, vol. 31, no. 18, pp. 4333-4342, 2008.

[9] R. Pighi and R. Raheli, "On multicarrier signal transmission for high-voltage power lines," in Proceedings of the 9th International Symposium on Power Line Communications and Its Applications (ISPLC '05), pp. 32-36, April 2005.

[10] DLC+VIT4IP, D1.2: Overall system architecture design DLC system architecture. FP7 Integrated Project No 247750, Jun. 2010.

[11] K. Dostert, Powerline Communications, Prentice-Hall, Upper Saddle River, NJ, 2001.

[12] N. Suljanović, A. Mujčić, M. Zajc, and J. F. Tasič, "Computation of high-frequency and time characteristics of corona noise on HV power line," IEEE Transactions on Power Delivery, vol. 20, no. 1, pp. 71-79, 2005.

[13] N. Suljanović, A. Mujčić, M. Zajc, and J. F. Tasič, "Integrated communication model of the HV power-line channel," in Proceedings of the IEEE International Symppsium on Power Line Communications and Its Applications, pp. 79-84, Zaragoza, Spain, 2004.

[14] N. Suljanović, A. Mujčić, M. Zajc, and J. F. Tasič, "Approximate computation of high-frequency characteristics for power line with horizontal disposition and middle-phase to ground coupling," Electric Power Systems Research, vol. 69, no. 1, pp. 17-24, 2004.

[15] U. A. Bakshi and M. V. Bakshi, Generation, Transmission and Distribution, Technical Publications Pune, Pune, India, 2001.

[16] J. C. de Sosa, Analysis and Design of High-Voltage Transmission Lines, iUniverse Incorporated, Bloomington, Ind, USA, 2010.

[17] N. M. Moyo, N. B. Ijumba, and A. C. Britten, "Investigations on the noise generation phenomena in the PLC system of a long HVDC line," in Proceedings of the International Conference on Power System Technology, pp. 953-957, Kunming, China, October 2002.

[18] N. Suljanović, A. Mujčić, M. Zajc, and J. F. Tasič, "Corona noise characteristics in high voltage PLC channel," in Proceedings of the IEEE International Conference on Industrial Technology, pp. 1036-1039, Maribor, Slovenia, December 2003.

[19] A. Mujčić, N. Suljanović, M. Zajc, and J. F. Tasič, "Design of channel coding methods in HV PLC communications," in Proceedings of the IEEE International Symposium on Power Line Communications and Its Applications, pp. 379-384, Zaragoza, Spain, 2004.

[20] A. Mujčić, N. Suljanović, M. Zajc, and J. F. Tasič, "High-voltage PLC roles in packet-switching networks of power utilities," in Proceedings of the IEEE International Symposium on Power Line Communications and Its Applications (ISPLC '07), pp. 204209, Pisa, Italy, March 2007.

[21] A. G. Lazaropoulos and P. G. Cottis, "Transmission characteristics of overhead medium-voltage power-line communication channels," IEEE Transactions on Power Delivery, vol. 24, no. 3, pp. 1164-1173, 2009.

[22] A. G. Lazaropoulos and P. G. Cottis, "Capacity of overhead medium voltage power line communication channels," IEEE Transactions on Power Delivery, vol. 25, no. 2, pp. 723-733, 2010.
[23] A. G. Lazaropoulos and P. G. Cottis, "Broadband transmission via underground medium-voltage power lines-part I: transmission characteristics," IEEE Transactions on Power Delivery, vol. 25, no. 4, pp. 2414-2424, 2010.

[24] A. G. Lazaropoulos and P. G. Cottis, "Broadband transmission via underground medium-voltage power lines-part II: capacity," IEEE Transactions on Power Delivery, vol. 25, no. 4, pp. 2425-2434, 2010.

[25] A. G. Lazaropoulos, "Towards broadband over power lines systems integration: transmission characteristics of underground low-voltage distribution power lines," Progress in Electromagnetics Research B, vol. 39, pp. 89-114, 2012.

[26] M. Gebhardt, F. Weinmann, and K. Dostert, "Physical and regulatory constraints for communication over the power supply grid," IEEE Communications Magazine, vol. 41, no. 5, pp. 84-90, 2003.

[27] P. S. Henry, "Interference characteristics of broadband power line communication systems using aerial medium voltage wires," IEEE Communications Magazine, vol. 43, no. 4, pp. 9298, 2005.

[28] S. Liu and L. J. Greenstein, "Emission characteristics and interference constraint of overhead medium-voltage Broadband Power Line (BPL) systems," in Proceedings of the IEEE Global Telecommunications Conference (GLOBECOM '08), pp. 29212925, December 2008.

[29] M. Götz, M. Rapp, and K. Dostert, "Power line channel characteristics and their effect on communication system design," IEEE Communications Magazine, vol. 42, no. 4, pp. 78-86, 2004.

[30] D. Fenton and P. Brown, "Some aspects of benchmarking high frequency radiated emissions from wireline communications systems in the near and far fields," in Proceedings of the Symposium on Power Line Communications and Its Applications, pp. 161-167, Malmö, Sweden, 2001.

[31] D. Fenton and P. Brown, "Modelling cumulative high frequency radiated interference from power line communication systems," in Proceedings of the Conference on Power Line Communications and Its Applications, Athens, Greece, 2002.

[32] M. Zimmermann and K. Dostert, "A multipath model for the powerline channel," IEEE Transactions on Communications, vol. 50, no. 4, pp. 553-559, 2002.

[33] S. Galli and O. Logvinov, "Recent developments in the standardization of power line communications within the IEEE," IEEE Communications Magazine, vol. 46, no. 7, pp. 6471, 2008.

[34] A. M. Tonello and F. Pecile, "Efficient architectures for multiuser FMT systems and application to power line communications," IEEE Transactions on Communications, vol. 57, no. 5, pp. 1275-1279, 2009.

[35] F. Versolatto and A. M. Tonello, "An MTL theory approach for the simulation of MIMO power-line communication channels," IEEE Transactions on Power Delivery, vol. 26, no. 3, pp. 1710-1717, 2011.

[36] T. Sartenaer, Multiuser communications over frequency selective wired channels and applications to the powerline access network [Ph.D. dissertation], Université catholique de Louvain, Louvain-la-Neuve, Belgium, 2004.

[37] S. Galli, A. Scaglione, and Z. Wang, "For the grid and through the grid: the role of power line communications in the smart grid," Proceedings of the IEEE, vol. 99, no. 6, pp. 998-1027, 2011.

[38] P. Amirshahi and M. Kavehrad, "High-frequency characteristics of overhead multiconductor power lines for broadband 
communications," IEEE Journal on Selected Areas in Communications, vol. 24, no. 7, pp. 1292-1302, 2006.

[39] T. Calliacoudas and F. Issa, "Multiconductor transmission lines and cables solver, an efficient simulation tool for plc channel networks development," in Proceedings of the Conference on Power Line Communications and Its Applications, Athens, Greece, 2002.

[40] S. Galli and T. C. Banwell, "A deterministic frequency-domain model for the indoor power line transfer function," IEEE Journal on Selected Areas in Communications, vol. 24, no. 7, pp. 1304-1315, 2006.

[41] S. Galli and T. Banwell, "A novel approach to the modeling of the indoor power line channel-part II: transfer function and its properties," IEEE Transactions on Power Delivery, vol. 20, no. 3, pp. 1869-1878, 2005.

[42] T. Sartenaer and P. Delogne, "Deterministic modeling of the (shielded) outdoor power line channel based on the Multiconductor Transmission Line equations," IEEE Journal on Selected Areas in Communications, vol. 24, no. 7, pp. 12771290, 2006.

[43] T. Sartenaer and P. Delogne, "Powerline cables modelling for broadband communications," in Proceedings of the IEEE International Conference on Power Line Communications and Its Applications, pp. 331-337, Malmö, Sweden, April 2001.

[44] C. R. Paul, Analysis of Multiconductor Transmission Lines, Wiley, New York, NY, USA, 1994.

[45] J. A. B. Faria, Multiconductor Transmission-Line Structures: Modal Analysis Techniques, Wiley, New York, NY, USA, 1994.

[46] A. Pérez, A. M. Sánchez, J. R. Regué et al., "Circuital and modal characterization of the power-line network in the PLC band," IEEE Transactions on Power Delivery, vol. 24, no. 3, pp. 11821189, 2009.

[47] H. Meng, S. Chen, Y. L. Guan et al., "Modeling of transfer characteristics for the broadband power line communication channel," IEEE Transactions on Power Delivery, vol. 19, no. 3, pp. 1057-1064, 2004.

[48] P. Amirshahi, Broadband access and home networking through powerline networks [Ph.D. dissertation], Pennsylvania State University, University Park, Pa, USA, 2006.

[49] J. Kabouris and G. C. Contaxis, "Electrical network optimization," in Encyclopedia of Life Support Systems (EOLSS), vol. 2 of Exergy, Energy System Analysis and Optimization, 2007.

[50] J. Kuffel, E. Kuffel, and W. S. Zaengl, High-Voltage Engineering Fundamentals, Butterworth-Heinemann, Woburn, UK, 2001.

[51] N. Suljanović, A. Mujčić, M. Zajc, and J. F. Tasič, "Highfrequency characteristics of high-voltage power line," in Proceedings of the IEEE International Conference on Computer as a Tool, pp. 310-314, Ljubljana, Slovenia, 2003.

[52] A. Mujčić, N. Suljanović, M. Zajc, and J. F. Tasič, "Influence of data frame duration on performance of BITCM system over HV power line," in Proceedings of the Joint 1st Workshop on Mobile Future and Symposium on Trends in Communications, pp. 150-153, Bratislava, Slovakia, October 2004.

[53] W. De Villiers, J. H. Cloete, and R. Herman, "The feasibility of ampacity control on HV transmission lines using the PLC system," in Proceedings of the 6th AFRICON Conference in Africa-Electrotechnological Services For Africa, pp. 865-870, George, South Africa, October 2002.

[54] M. Zajc, N. Suljanović, A. Mujčić, and J. F. Tasič, "Frequency characteristics measurement of overhead high-voltage powerline in low radio-frequency range," IEEE Transactions on Power Delivery, vol. 22, no. 4, pp. 2142-2149, 2007.

[55] M. Z. A. Ab Kadir, J. Sardi, W. F. Wan Ahmad, H. Hizam, and J. Jasni, "Evaluation of a $132 \mathrm{kV}$ transmission line performance via transient modelling approach," European Journal of Scientific Research, vol. 29, no. 4, pp. 533-539, 2009.

[56] R. K. Z. Sahbudin, S. A. Fauzi, S. Hitam, and M. Mokhtar, "Investigation of electrical potential and electromagnetic field for overhead high voltage power lines in Malaysia," Journal of Applied Sciences, vol. 10, no. 22, pp. 2862-2868, 2010.

[57] Tenaga Nasional Berhad (TNB), "Variation Orders in Transmission Projects of Tenaga Nasional Berhad," Tech. Rep., Malaysia, 2006.

[58] M. D'Amore and M. S. Sarto, “A new formulation of lossy ground return parameters for transient analysis of multiconductor dissipative lines," IEEE Transactions on Power Delivery, vol. 12, no. 1, pp. 303-309, 1997.

[59] P. Amirshahi and M. Kavehrad, "Medium voltage overhead power-line broadband communications; transmission capacity and electromagnetic interference," in Proceedings of the 9th International Symposium on Power Line Communications and Its Applications (ISPLC '05), pp. 2-6, April 2005.

[60] M. D'Amore and M. S. Sarto, "Simulation models of a dissipative transmission line above a lossy ground for a widefrequency range-part I: single conductor configuration," IEEE Transactions on Electromagnetic Compatibility, vol. 38, no. 2, pp. 127-138, 1996.

[61] M. D'Amore and M. S. Sarto, "Simulation models of a dissipative transmission line above a lossy ground for a widefrequency range-part II: multiconductor configuration," IEEE Transactions on Electromagnetic Compatibility, vol. 38, no. 2, pp. 139-149, 1996.

[62] J. Anatory and N. Theethayi, "On the efficacy of using ground return in the broadband power-line communications-a transmission-line analysis," IEEE Transactions on Power Delivery, vol. 23, no. 1, pp. 132-139, 2008.

[63] J. R. Carson, "Wave propagation in overhead wires with ground return,” Bell System Technical Journal, vol. 5, pp. 539$554,1926$.

[64] H. Kikuchi, "Wave propagation along an infinite wire above ground at high frequencies," Electrotechnical Journal of Japan, vol. 2, pp. 73-78, 1956.

[65] H. Kikuchi, "On the transition form a ground return circuit to a surface waveguide," in Proceedings of the Congress on Ultrahigh Frequency Circuits Antennas, pp. 39-45, Paris, France, October 1957.

[66] F. Issa, D. Chaffanjon, E. P. de la Bâthie, and A. Pacaud, "An efficient tool for modal analysis transmission lines for PLC networks development," in Proceedings of the IEEE International Conference on Power Line Communications and Its Applications, Athens, Greece, 2002.

[67] W. de Villiers, J. H. Cloete, L. M. Wedepohl, and A. Burger, "Real-time sag monitoring system for high-voltage overhead transmission lines based on power-line carrier signal behavior," IEEE Transactions on Power Delivery, vol. 23, no. 1, pp. 389-395, 2008.

[68] S. G. Lodwig and C. C. Schuetz, "Coupling to control cables in HV substations," in Proceedings of the International Symposium on Electromagnetic Compatibility, pp. 249-253, Montreal, Canada, August 2001.

[69] D. Anastasiadou and T. Antonakopoulos, "Multipath characterization of indoor power-line networks," IEEE Transactions on Power Delivery, vol. 20, no. 1, pp. 90-99, 2005.

[70] S. Barmada, A. Musolino, and M. Raugi, "Innovative model for time-varying power line communication channel response evaluation," IEEE Journal on Selected Areas in Communications, vol. 24, no. 7, pp. 1317-1325, 2006. 
[71] J. A. Dobrowolski, Microwave Network Design Using the Scattering Matrix, Artech House, Norwood, Mass, USA, 2010.

[72] R. Mavaddat, Network Scattering Parameters. Advanced Series in Circuits and Systems, World Scientific Publishing Company, River Edge, NJ, USA, 2nd edition, 1996.

[73] G. Gonzalez, Microwave Transistor Amplifiers: Analysis and Design, Prentice-Hall, Upper Saddle River, NJ, USA, 2nd edition, 1996.

[74] S. Galli, A. Scaglione, and K. Dostert, "Broadband is power: internet access through the power line network," IEEE Communications Magazine, vol. 41, no. 5, pp. 82-83, 2003.

[75] H. A. Latchman and L. W. Yonge, "Power line local area networking," IEEE Communications Magazine, vol. 41, no. 4, pp. 32-33, 2003.

[76] E. Fortunato, A. Garibbo, and L. Petrolino, "An experimental system for digital power line communications over high voltage electric power lines-field trials and obtained results," in Proceedings of the IEEE International Symposium Power Line Communications and Its Applications, pp. 26-31, Kyoto, Japan, 2003.

[77] J. Anatory, N. Theethayi, and R. Thottappillil, "Powerline communication channel model for interconnected networks - part II: multiconductor system," IEEE Transactions on Power Delivery, vol. 24, no. 1, pp. 124-128, 2009.

[78] H. Philipps, "Modelling of powerline communications channels," in Proceedings of the International Symposium on Power Line Communications and Its Applications, pp. 14-21, Lancaster, UK, 1999.

[79] J. Anatory, N. Theethayi, R. Thottappillil, M. M. Kissaka, and N. H. Mvungi, "The influence of load impedance, line length, and branches on underground cable power-line communications (PLC) systems," IEEE Transactions on Power Delivery, vol. 23, no. 1, pp. 180-187, 2008.

[80] J. Anatory, N. Theethayi, R. Thottappillil, M. Kissaka, and N. Mvungi, "The effects of load impedance, line length, and branches in typical low-voltage channels of the BPLC systems of developing countries: transmission-line analyses," IEEE Transactions on Power Delivery, vol. 24, no. 2, pp. 621-629, 2009.

[81] OPERA1, D5: Pathloss as a function of frequency, distance and network topology for various LV and MV European powerline networks. IST Integrated Project No 507667, 2005.

[82] M. Kuhn, S. Berger, I. Hammerström, and A. Wittneben, "Power line enhanced cooperative wireless communications," IEEE Journal on Selected Areas in Communications, vol. 24, no. 7, pp. 1401-1410, 2006.

[83] R. Aquilué, I. Gutierrez, J. L. Pijoan, and G. Sánchez, "Highvoltage multicarrier spread-spectrum system field test," IEEE Transactions on Power Delivery, vol. 24, no. 3, pp. 1112-1121, 2009.

[84] A. M. Sarafi, A. E. Drougas, and P. G. Cottis, "Crosslayer resource allocation in medium-voltage broadband over power-line networks," IEEE Transactions on Power Delivery, vol. 27, no. 4, pp. 2247-2254. 

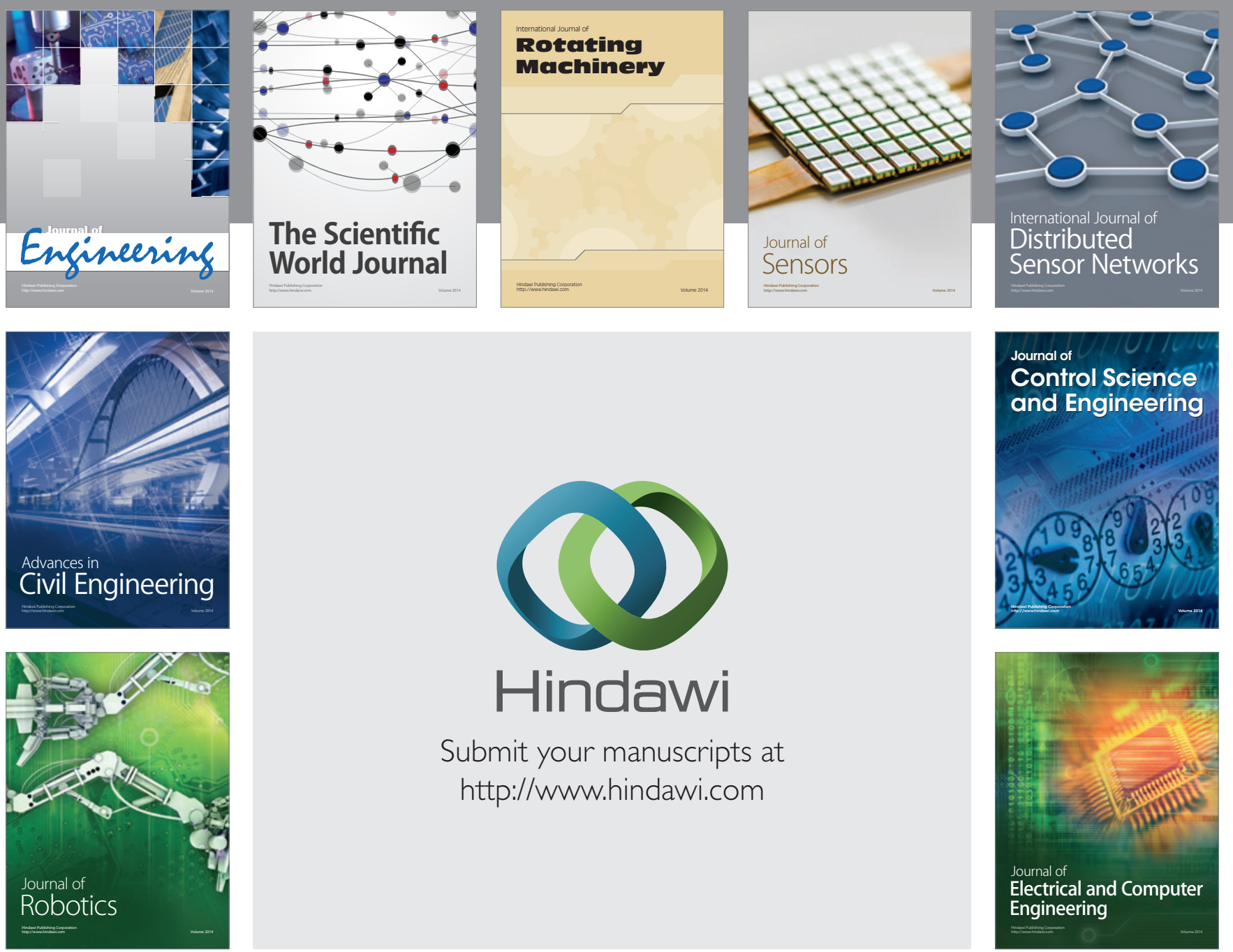

Submit your manuscripts at

http://www.hindawi.com
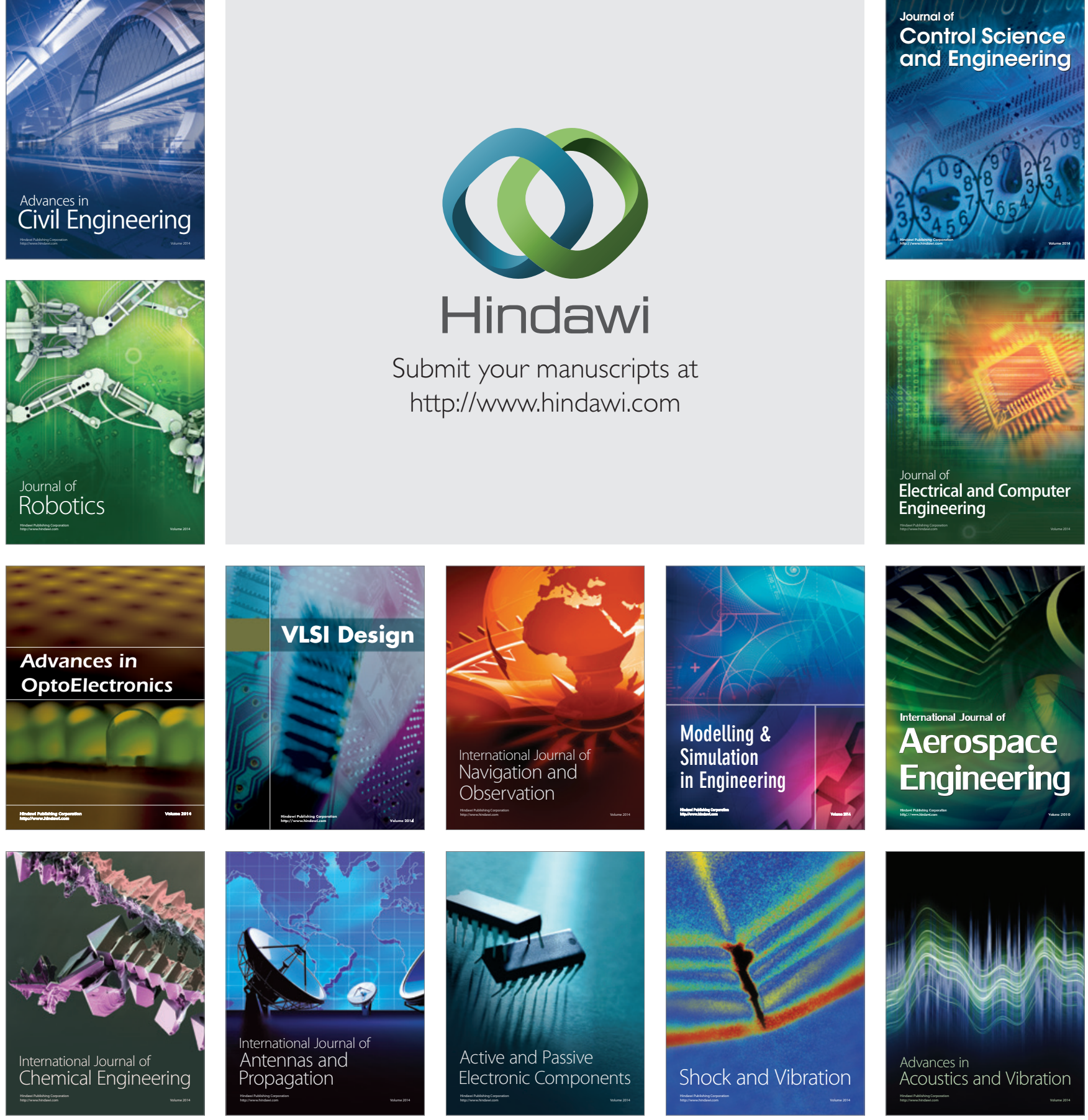